\title{
TEOLOGIA, ORDINI RELIGIOSI E RAPPORTI POLITICI: LA QUESTIONE DELL'IMMACOLATA CONCEZIONE DI MARIA TRA ROMA E MADRID (1614-1663)
}

\author{
POR
}

\section{PAOLO BROGGIO}

Dipartimento di Studi Umanistici

Università degli Studi Roma Tre

RESUMEN: El artículo analiza los primeros años de las polémicas doctrinales alrededor de la definición dogmática de la Inmaculada Concepción de la Virgen desde el punto de vista de las relaciones entre la Santa Sede y la Monarquía española, gestionadas por el nuncio Antonio Caetani. Sobre la base de la correspondencia epistolar del nuncio con la Curia romana se evidencia la voluntad, por parte de Roma, de evitar los "escándalos" de los alborotos populares y de buscar una mediación entre las órdenes religiosas en lucha (dominicos, franciscanos y jesuitas). Estas polémicas fueron una ocasión más, para la Monarquía hispana de Felipe III (y luego de Felipe IV), para afirmar su propio derecho de intervención en asuntos doctrinales y eclesiásticos en virtud de su prerogativa de defensa de la Iglesia de Roma.

PALABRAS CLAVES: Teología, Contrarreforma, Inmaculada Concepción, Curia romana, Monarquía española, Relaciones internacionales.

THEOLOGY, RELIGIOUS ORDERS AND POLITICAL RELATIONS: THE QUESTION OF THE IMMACULATE CONCEPTION OF MARY BETWEEN MADRID AND ROME

ABSTRACT: The article analyses the first moments of the doctrinal controversy about the dogmatic definition of the Immaculate Conception of Mary from the point of view of the relationships, managed by nuncio Antonio Caetani, between the Holy See and the Spanish Monarchy. Through the correspondence between the nuncio and the Roman Curia it is possible to appreciate Papacy's will to avoid public "scandals" and to look for a mediation among conflicting religious orders (Dominicans, Franciscans and Jesuits). The controversy represented for the Spanish Monarchy of Philip III (and, later, of Philip IV) a further occasion to affirm its

Dedico questo articolo alla memoria di Juan Luis Castellano Castellano.

Abbreviazioni: ACDF: Archivio della Congregazione per la Dottrina della Fede (Roma); AGOFM: Archivum Generale Ordinis Fratrum Minorum (Roma); AMAE: Archivo del Ministerio de Asuntos Exteriores (Madrid); ASV: Archivio Segreto Vaticano; BUG: Biblioteca Universitaria de Granada. 
right of intervention in doctrinal and ecclesiastical matters on the basis of its prerogative to defend the Roman Church.

KEY WORDS: Theology, Counterreformation, Immaculate Conception, Roman Curia, Spanish Monarchy, International relations.

$\begin{array}{ll}\text { Recibido/Received } & 02-08-2011 \\ \text { Acceptado/Accepted } & 20-11-2012\end{array}$

\section{COSTRUZIONE DI UNA MONARCHIA CONFESSIONALE E RAPPORTI ISPANO-PONTIFICI}

In un suo recente intervento pubblicato in Italia Juan Luis Castellano, nel descrivere il processo di sacralizzazione della Monarchia spagnola in età moderna, parallelo a quello di assolutizzazione, cita un passo dello scrittore politico Gregorio López de Madera, che alla fine del Cinquecento affermava che «tutto ciò che è limpido e senza macchia di eresia o di errori nella fede, tutta la sovranità che può veramente dirsi cattolica, è la corona e la monarchia di Spagna». ${ }^{1}$ A partire dal Cinquecento due divennero gli assi principali dell'azione politica della Monarchia spagnola: la diffusione dei buoni costumi nel corpo sociale e la difesa della purezza della fede, obiettivo per il quale i re cattolici potevano disporre già dalla seconda metà del XV secolo, grazie a una speciale delega pontificia, di uno strumento straordinario come I'Inquisizione. La precettistica politica prodotta negli anni compresi tra il 1580 e il 1620 rappresenta un'eccellente indicatore della cristallizzazione di un'ideologia del potere che faceva della difesa della fede e della Chiesa di Roma il perno dell'azione politica dei sovrani asburgici, e della sacralizzazione del figura del re il presupposto della cieca obbedienza al potere costituito, al sovrano, ai suoi ministri (in quanto riflessi della volontà divina). Un concetto, quest'ultimo, ribadito incessantemente dai pulpiti e nei confessionali. L'idea della purezza della fede, dell'esenzione del cattolicesimo spagnolo dalla macchia dell'eresia, non poteva del resto non tradursi nell'esclusione dalla vita politica della Monarchia di tutti coloro che non potessero vantare un pedigree inconfutabilmente cristiano viejo (pensiamo agli statuti di limpieza de sangre) e ancor più tragicamente - nel rigetto delle minoranze religiose (gli ebrei, cacciati nel 1492, e i moriscos, espulsi definitivamente dalla penisola a partire dal 1609) e nella sistematica persecuzione di giudaizzanti e islamizzanti.

Ma i contrasti tra la Monarchia spagnola e Roma, oltre a conoscere variazioni di rilievo a seconda delle personalità di singoli pontefici e sovrani, non furono semplicemente «le contraddizioni inevitabili di una monarchia che era fino in fondo confessionale», ${ }^{2}$ come scrive ancora Castellano. Più che di contraddizioni si dovrebbe piuttosto parlare di prodotto logico e conseguente, consustanziale a una azione politica che ancor più che alla difesa della Chiesa cattolica, apostolica e romana mirava al raggiungimento di una posizione tutoria nei confronti del centro della cattolicità, e questo proprio nel momento in cui l'eclissi alla corte di Madrid del partito "castiglianista" autorizzava i contemporanei (e autorizza anche alcuni storici

${ }^{1}$ Cit. in Castellano, J.L. 2008. "La monarchia spagnola come paradigma di una monarchia confessionale", Dimensioni e problemi della ricerca storica 172, dall'opera López de Madera, G. 1597. Excelencias de la Monarquía y reyno de España: 82 Valladolid: Imprenta de Diego Fernández de Córdova.

2 Ibidem, p. 179. 
odierni) ${ }^{3}$ a parlare perfino di sudditanza della Spagna di Filippo III nei confronti di pontificati autorevoli come quelli di Clemente VIII Aldobrandini e di Paolo $\mathrm{V}$ Borghese. $^{4}$

Tale rilievo mi sembra particolarmente pertinente per ciò che attiene al campo dottrinale, in cui la diplomazia spagnola fu particolarmente attiva, negli anni a cavallo tra Cinque e Seicento, allo scopo di conquistarsi un ruolo che non fosse di mera cassa di risonanza (e braccio armato) di una - peraltro - non ben identificabile ortodossia romana. I contrasti di carattere politico che accompagnarono lo svolgimento della controversia de auxiliis divinae gratiae, soprattutto a partire dall'avocazione romana del 1594, non furono che l'inizio di un percorso di affermazione, da parte della Monarchia spagnola degli Asburgo, di un legittimo diritto di intervento in questioni di carattere teologico-dottrinale e, di conseguenza, nelle questioni religiose ed ecclesiastiche tout court. ${ }^{5}$ In tale contesto la questione del ruolo degli ordini religiosi e della loro influenza a corte è evidentemente centrale, ed è proprio su questo terreno che vorrei svolgere le mie considerazioni, al fine di mettere in rilievo la scarsa adeguatezza di schemi interpretativi tanto lineari quanto spesso non in grado di cogliere la complessità dei reticoli di interessi, aderenze, protezioni, in vario modo collegati a questioni "alte", che sono alla base della costruzione delle ideologie del potere $\mathrm{e}$ in cui l'elemento idiomatico risulta fondamentale. ${ }^{6}$

I dibattiti intorno alla dottrina dell'Immacolata Concezione ci offrono uno spaccato non solo della storia delle dottrine teologiche e della religiosità spagnola del primo Seicento ma anche un ritratto, per certi aspetti inedito, del processo di costruzione politico-istituzionale e identitaria dell'Europa cattolica, e della Monarchia ispanica in modo particolare, nella prima età moderna. II riconoscimento ufficiale e definitivo della dottrina del concepimento senza macchia di Maria costituiva un tassello di ciò che Adriano Prosperi ha indicato come la «costruzione di una religione nazionale e di uno specifico rapporto tra sudditi e sovrano». ${ }^{7}$ Non è un caso infatti - sottolinea Prosperi - che la questione dell'Immacolata abbia preso una direzione del tutto particolare proprio in Spagna e proprio agli inizi del Seicento: si tratta del trionfo di una religione dell'onore maschile e della purezza femminile che si fa specchio di una vera e propria ossessione nei confronti dell'eterodossia

${ }^{3}$ Mi riferisco soprattutto a Martínez Millán J., Visceglia M.A. (eds.) 2008. La Monarquía de Felipe III: la casa del rey, Madrid, Fundación MAPFRE.

4 Una eccellente analisi dei rapporti ispano-pontifici tra Cinque e Seicento è offerta da Visceglia, M.A. 2009. Roma e papale e Spagna. Diplomatici, nobili e religiosi tra due corti, Roma, Bulzoni.

${ }^{5}$ Ho sviluppato questo tema in Broggio, P. 2009. La teologia e la politica. Controversie dottrinali, Curia romana e Monarchia spagnola tra Cinque e Seicento, Firenze, Leo S. Olschki.

6 Sulla necessità di cogliere il nesso tra la micropolitica dei legami clientelari e la macropolitica delle grandi questioni nei rapporti tra Roma e Madrid richiama l'attenzione Visceglia, M.A. 2007. "Roma e la monarchia Cattolica nell'età dell'egemonia spagnola in Italia: un bilancio storiografico", in: C.J. Hernando Sánchez (ed.), Roma y España. Un crisol de la cultura europea en la Edad Moderna, Madrid, 2007, 72.

7 Prosperi, A. 2006. "L'Immacolata e Siviglia e la fondazione sacra della monarchia spagnola”. Studi Storici XLVII: 481-510 [482]. Sull'Immacolata a Siviglia cfr. anche Ollero Pina, J.A. 2003. "Sine labe concepta: conflictos eclesiásticos e ideológicos en la Sevilla de principios del siglo XVII", in C.A. González Sánchez, E. Vila Vilar (eds.), Grafías del Imaginario. Representaciones culturales en España y América (siglos XVI-XVIII): 301-335. México, Fondo de Cultura Económica. 
religiosa e dei disegni politici di una Monarchia cattolica che si vuole presentare su scala continentale e planetaria come immune dalla macchia disonorante ed ereditaria dell'eresia, una colpa che si trasmette di padre in figlio. Né, d'altronde, bisogna sottovalutare il contesto specifico in cui la controversia sull'Immacolata si accese, quell'Andalusia in pieno fermento sociale, politico e religioso, duramente provata dall'espulsione dei moriscos ed eccitata dal ritrovamento dei libri plumbei di Granada, che faticava ad adattarsi ai nuovi canoni devozionali dettati dalla Controriforma e in cui il tradizionale culto mariano si inscriveva in quegli anni in un contesto contrassegnato dal «moltiplicarsi delle manifestazioni devote che presero i caratteri della festa barocca [e che] crearono un clima fortemente emotivo in cui si inserì un'intensa attività di propaganda attraverso le rappresentazioni, le immagini, gli scritti»s. ${ }^{8}$

Si vuole in questo intervento analizzare la controversia sull'Immacolata Concezione di Maria, così come si è sviluppata in Spagna a partire dal 1614, dal punto di vista dei rapporti tra Madrid e la Santa Sede, gestiti sin dalle prime avvisaglie dal nunzio Antonio Caetani. Sarà utilizzata soprattutto la ricca documentazione inedita custodita presso l'Archivio della Congregazione per la Dottrina della Fede, specchio prezioso degli scambi diplomatici intercorsi tra il Sant'Ufficio, il nunzio pontificio in Spagna e la corte madrilena a partire dal 1615.

\section{PRIMI BAGLIORI. GLI INIZI ANDALUSI DELLA CONTROVERSIA} SULL'IMMACOLATA CONCEZIONE DI MARIA

Tutto ebbe inizio in Andalusia. Ma come in ogni controversia che si rispetti l'individuazione della prima scintilla degli scontri sull'Immacolata è anch'essa materia di controversia; ognuna delle parti in lite differisce infatti circa il tempo, il luogo e la responsabilità dell'evento che scatenò la contesa. II nunzio Antonio Caetani avrebbe scritto nel settembre del 1615: «Del certo da che parte sia veramente venuto il primo origine del disordine non sarà così facile il poter verificarlo», aggiungendo però subito dopo che «il più certo è che in Cordova cominciò il primo principio, i domenicani dicono per occasione data da un teologo secolare, et i contrarii da un padre, che volse vendicare in pulpito lo sdegno con troppa collera». ${ }^{9}$ Stessa versione sarebbe del resto stata accolta nella ricostruzione ufficiale di parte spagnola delle polemiche sull'Immacolata, se è vero che secondo la Relación histórico-theológico-política de lo sucedido en el santo negocio de la Concepción inmaculada de la Virgen sanctísima (1615-1672), compilata a partire dagli anni Cinquanta per volere di Filippo IV, la scaturigine degli scontri viene senza esitazioni individuata nella richiesta da parte di un canonico della cattedrale di Cordova, Álvaro Pizaño de Palacios, di far predicare per la festa dell'Immacolata del 1612 un frate domenicano, richiesta che si sarebbe ripetuta l'anno successivo all'indirizzo del padre Cristóbal de Torres. Pizaño sarebbe poi passato a Siviglia, dove non ebbe difficoltà nel far ardere il fuoco degli scontri tra macolisti e immacolisti. ${ }^{10}$ Secondo altre fonti, invece, tutto avrebbe avuto inizio a Siviglia l'8

${ }^{8}$ Cfr. Visceglia, M.A. 2009: 255.

${ }^{9}$ II nunzio Antonio Caetani al cardinale Giangarzia Millini, 27 settembre 1615, ACDF, S.O., St. St. M 6 a, f. $27 r-v$.

${ }^{10} \mathrm{La}$ ponderosa ricostruzione storica, ancora manoscritta ed estremamente interessante, della controversia sull'Immacolata, assolve il canonico cordovese Pizaño dall'accusa di essere il fomentatore degli scontri andalusi sull'Immacolata, un'accusa che gli era stata mossa dal confessore regio Luis de Aliaga. Quest'ultimo, nel 1618, avrebbe consegnato a Filippo III un memoriale in cui affermava che il principio dei disordini era da attribuirsi a Pizaño, che aveva predicato la Concezione 
settembre 1613, in un convento di suore domenicane, quando un predicatore dello stesso ordine ebbe l'ardire di dichiararsi contrario all'immacolato concepimento di Maria. Siviglia, città in cui il culto mariano era particolarmente vivo, non era evidentemente il luogo adatto per una simile dichiarazione. Diffusasi la notizia sempre stando a questa ricostruzione - la folla dei fedeli mise in atto una vera e propria sommossa, marciando compatta in direzione del convento cui apparteneva il predicatore con l'intenzione di "farsi giustizia". In reazione a quella che veniva avvertita come un'oltraggiosa predica nei mesi successivi vennero organizzate da francescani e gesuiti numerose manifestazioni di devozione - novene, ottavari, processioni, ecc. - finalizzate all'eccitamento della pietà popolare mariana. La controversia prese da subito un carattere marcatamente popolare. Ciò che i pontefici, sin da Sisto IV (1471-1484), avevano cercato di evitare, ossia il coinvolgimento del popolo minuto, anche attraverso il divieto della pubblicazione di opere in lingua volgare sulla questione, si produsse così a Siviglia generando veri e propri tumulti e, come si diceva all'epoca, enorme "scandalo".

La disputa sull'Immacolata Concezione può essere letta anche solo come un ennesimo contrasto di natura dottrinale tra ordini religiosi dopo gli anni delle aspre e profonde polemiche sulla questione della grazia che avevano costretto papa Clemente VIII ad avocare a sé il giudizio sulla controversia tra gesuiti e domenicani; una questione chiusa in maniera salomonica da Paolo $\mathrm{V}$ nell'agosto del 1607 ma che aveva conosciuto considerevoli strascichi negli anni immediatamente successivi, con episodi di grave tensione diplomatica tra Roma e Madrid. ${ }^{11}$ Si tratta di uno schema interpretativo che la storiografia domenicana non ha mai cessato di utilizzare; ancora agli inizi degli anni trenta del Novecento Maximiliano Canal scriveva, a commento di uno dei tanti sonetti antidomenicani che circolarono a Siviglia dopo il 1613, che:

Mas no eran los franciscanos quienes propiamente indisponían al pueblo contra los dominicos. Aunque dé grima descubrir estas cosas, sin embargo como la historia es historia y hasta ahora se ha escrito, en lo que toca a este punto, con falta de documentación y sobra de parcialidad, es fuerza decir que en todo aquel alboroto tenían gran mano algunos padres de la Compañía. ${ }^{12}$

La continuità di motivi politici tra la disputa de auxiliis e la controversia sull'Immacolata è innegabile e forte, specie nei riflessi polemici che toccavano nel vivo il problema - assolutamente centrale - delle prerogative della Santa Sede,

della Vergine «ofensiva y desatentadamente»; «esto dice el padre confesor, pero la verdad es lo que queda dicho, y bien se deja entender, que el doctor Pizaño no fue el que comenzó, pues lo que dixo fue en defensa de la verdad del sentido de la fiesta contra quien avían predicado dos religiosos de santo Domingo, y assí ellos fueron los que dieron principio o casi en las rebueltas». Relación histórico-theológico-política de lo sucedido en el santo negocio de la Concepción inmaculada de la Virgen sanctísima (1615-1672), Primera parte de lo sucedido en tiempo del Rey Nuestro Señor Don Phelipe tercero, que Dios tiene, AMAE, Ms. 446.

${ }^{11}$ Cfr. Lopetegui, L. 1974. "Renovación y fracaso de las tentativas para obtener una definición dogmática en la Controversia de Auxiliis 1607-1614", Archivo Teológico Granadino 37: 4581.

${ }^{12}$ Canal M., 1932. "El p. Luis de Aliaga y las controversias teológicas de su tiempo, Archivum Fratrum Praedicatorum 1: 141. 
rispetto alla Monarchia spagnola e all'Inquisizione, nella soluzione delle controversie dottrinali. Ma negli inizi andalusi dello scontro sull'Immacolata c'è, a ben vedere, anche dell'altro: si avverte una sorta di rivolta generale, tra i laici e nelle altre famiglie religiose, contro l'ordine domenicano che va attentamente considerata e contestualizzata se si vuole giungere a una migliore intelligenza delle vicende che si stanno considerando. A chiedere a Roma un deciso intervento contro il comportamento dei domenicani, infatti, non sono solo i loro diretti concorrenti, i francescani e i gesuiti, o il principale sostenitore in loco della causa immacolista, l'arcivescovo di Siviglia Pedro de Castro y Quiñones, nominato nel 1610 dopo aver guidato la sede granadina in anni fondamentali per la vicenda delle lamine plumbee. $^{13}$ Gli interessi in gioco erano molteplici e a muoversi repentinamente contro i domenicani furono interi organi rappresentativi di città andaluse, gelose della propria devozione immacolista, istituzioni educative gestite da vari ordini religiosi, università che imponevano ormai da tempo il giuramento immacolista ai propri membri, così come conventi intitolati all'Immacolata che avrebbero subito un danno grave - e non solo d'immagine - in caso di vittoria delle tesi macoliste.

II 14 luglio 1614, un anno prima che il nunzio Caetani desse inizio al proprio tentativo di mediazione, il Collegio imperiale di Granada si indirizzava a Roma per far presenti le insidie insite in una situazione in cui i domenicani avevano osato mettere in dubbio la verità dell'immacolato concepimento della Vergine Maria, una verità che in Spagna era già di fatto considerata "di fede", su cui poggiavano non solo la devozione dei fedeli ma anche intitolazioni di conventi, regole di ordini monastici, consolidate tradizioni universitarie:

La institución de la fiesta, de la Inmaculada concepción de la Virgen María señora nuestra (santísimo Padre) desde los tiempos de Sisto quarto, de felice recordación, cuya solemnidad, ochocientos años, había que la comenzó, a celebrar, la Iglesia griega; la confirmazión de la regla de las hermanas de la Concepción, por los santos padres Alexandro sexto, Julio segundo, y Leon dézimo, de que hay muchos conventos en España, la suma piedad, y rara devoción, con que todas las iglesias, cathedrales y conventos de religiosos, de este rreino, celebran esta festividad, hasta las Universidades, en los grados que dan, obligan a quien los reciben a defender, este assumpto. Todo aquesto ha engrendado (sic) en los ánimos de casi todos los fieles tan grande devoción, con tan marabillosso augmento, que nadie se ha atrevido, por ella, y por el altecal, de quien toca ni en púlpitos, ni en cathedrales, a sentir lo contrario. ${ }^{14}$

La richiesta al pontefice era di chiarire al più presto, dichiarando «lo que se ha de leer, y predicar en esta materia». Ma molte altre città andaluse iniziarono

${ }^{13}$ Cfr. Martínez Medina, F.J., 2006. "Teólogos, religiosidad y magisterio en el Sacromonte de Granada. Los discursos inmaculistas de los libros plúmbeos", in A.L. Cortés Peña (ed.), Poder civil, Iglesia y sociedad en la edad moderna: 295-342. Granada: Universidad de Granada. Sulle complesse vicende delle lamine di Granada cfr. in generale Barrios Aguilera, M. García-Arenal (eds.), 2006. Los plomos del Sacromonte: invención y tesoro, Valencia-Granada-Zaragoza, Universidad de Valencia; García-Arenal, F. Rodríguez Mediano, 2010. Un Oriente español. Los Moriscos y el Sacromonte en tiempos de Contrarreforma, Madrid, Marcial Pons. In particolare sui rapporti con Roma cfr. Scaramella, P., 2008. "«Una materia gravissima, una enorme heresia». Granada, Roma e la controversia sugli apocrifi del Sacromonte". Rivista storica italiana CXX 3: 10031044.

\footnotetext{
${ }^{14}$ Memoriale del Collegio Imperiale di Granada, ACDF, S.O., St. st., M 6 a, f. 36r.
} 
tempestivamente - siamo nell'estate del 1615 - a rivolgersi a Roma affinché intervenisse per mettere a tacere ciò che veniva avvertito da tutti come lo scandaloso macolismo dei domenicani: Andújar, nella diocesi di Jaén, che di fronte al pontefice rivendicava, tra l'altro, una diretta ascendenza romana della sua primitiva evangelizzazione $^{15}$ e che ricordava al pontefice che i domenicani avevano intrapreso la propria campagna «con tal coraje y porfía que si Vuestra Santidad como padre de todos no interpone su autoridad se teme creçerán los inconvenientes»; ${ }^{16}$ Loja, nell'arcidiocesi di Granada, che chiedeva a Paolo V di «declarar la verdad cathólica deste punto, así por consuelo general de las almas como por quietud universal de la Iglesia»; ${ }^{17}$ Alcalá la Real (con il suo abate) e Vélez, nell'arcidiocesi di Granada; Lucena, nella diocesi di Cordova. Ma oltre alle città a intervenire furono, ad esempio, il monastero di San Jerónimo di Granada, il locale convento e collegio dell'ordine mercedario, il Collegio di Santa Caterina martire (collegato allo Studium granadino), l'abate e il capitolo cattedrale di Baza (arcidiocesi di Granada), il capitolo della cattedrale di Cordova, che indirizzò il proprio memoriale alla Congregazione dei vescovi e regolari. Quest'ultimo documento ci rivela particolari e circostanze interessanti per comprendere la dinamica dello scontro che si diffuse con grande rapidità in Andalusia, e dall'Andalusia a tutta la Spagna. II memoriale si riferisce infatti alla predica tenuta dal frate domenicano Cristóbal de Torres, del convento di San Pablo, in occasione della festività dell'Immacolata del 1614. II frate, ci rammenta il memoriale,

altre volte havea predicato detta predica con alcuna nota di libertà sopra la purità della Madonna, et per ovviare al scandalo, che poteva succedere si detto frate in detto giorno predicasse alcuni capitolari temendosi di quello, che havea da succedere, procurarono, tanto con monsignore vescovo, quanto con esso frate, che lasciassi detta predica, o almeno attendesse a non dire cosa, che fosse contra la festa, che si faceva, et celebrava la Chiesa romana sopra la purità della Concettione della Madonna, perché altrimente il popolo restarebbe scandalizzato. ${ }^{18}$

Il padre Torres non si mantenne entro i limiti della prudenza e la sua predica provocò la dura reazione del capitolo cattedrale e scandalo tra i fedeli. Alcuni studi parlano di Torres come di un frate domenicano che - su pressione del capitolo ricevette una reprimenda dal vescovo di Cordova, il confratello Diego de Mardones, già confessore di Filippo III e del duca di Lerma, ma che ciò nonostante perseverò nel proprio atteggiamento di aperta sfida nei confronti del capitolo cattedrale e dei devoti della città. ${ }^{19}$ A leggere invece questo memoriale l'impressione che si ricava è sensibilmente diversa: la decisione del prelato domenicano di scegliere religiosi del

15 «Córrele a esta ciudad de Andújar particular obligación de ser muy hiia de Vuestra Santidad y su sanctísima silla por haber recebido su fe y religión cathólica con la doctrina y sangre del sanctíssimo martyr san Euphrasio su obispo discípulo de los sanctos apóstoles san Pedro y san Pablo y enbiado por ellos a España»; il memoriale è firmato da Rodrigo de Valenzuela e dal licenciado Pedro de Carvájal in nome della città di Andújar. ACDF, S.O., St. st. M 6 a, f. 45v.

16 Idem.

${ }^{17}$ ACDF, S.O., St. st. M 6 a, f. 50v.

18 Ibidem, f. 5 r.

${ }^{19}$ Cfr. Vázquez Lesmes, R., "Pizaño de Palacios, un inmaculista maculado", Boletín de la Real Academia de Córdoba de ciencias, bellas letras y nobles artes: CXXIV (1993): 181-192. 
proprio ordine per predicare in occasione dell'Immacolata viene infatti presentata come una consuetudine (Mardones era vescovo di Cordova sin dal 1607), così come sembra non essere stata una novità la libertà con la quale tali predicatori avevano in passato affrontato la dottrina dell'Immacolata:

A monsignor vescovo cordubense tocca raccomandare la predica, che si ha da predicare nella Chiesa cordubense il giorno della Concettione della Madonna, et perché esso è della religione di san Domenico sempre la raccomanda a frati domenicani, di che l'anni passati vi è seguito grandissimo scandalo volendo detti predicatori con troppa libertà trattare sopra l'Immaculata conceptione della Madonna, di che non solo li capitolari et ecclesiastici ma anco secolari si sono grandemente scandalizzati, et acciò la devotione che il popolo ha verso la purità della Madonna, non si diminuisca ma più tosto cresca. $^{20}$

Ma allora perché solo quell'anno, e non prima, la predica di un domenicano nella cattedrale della città fu in grado di generare polemiche, disordini, proteste, scandalo, sia nel popolo che nelle principali istituzioni laiche ed ecclesiastiche della città? Se l'ennesima predica troppo licenziosa di Torres spinse l'intero capitolo della cattedrale di Cordova a rivolgersi a Roma il clima politico-religioso generale doveva essere evidentemente cambiato rispetto agli anni immediatamente precedenti. ${ }^{21} \mathrm{Se}$ ci si sposta poi a Siviglia l'impressione riceve una conferma: in una testimonianza rogata di fronte a un notaio e inviata a Roma dall'arcivescovo Castro allo scopo di inchiodare i domenicani alle loro responsabilità di perturbatori dell'ordine pubblico, si fa esplicitamente riferimento proprio a un repentino cambiamento di atteggiamento da parte dei domenicani sulla questione dell'Immacolata. II contesto contingente era un'ormai consolidata tradizione locale che vedeva i frati domenicani accompagnare i membri di una confraternita intitolata all'Immacolata nella processione del Giovedì santo. Nella settimana santa del 1614,

estando la cofradia de la limpia concepción de Nuestra Señora en immemorial posessión, de que los frailes de Regina angelorum orden de santo Domingo, la acompañen todos los jueves santos en la processión de deçeplina que los cofrades hacen. Este año de seiscientos y catorçe, no quisieron salir los dichos padres, porque llamavan novedad, que en el palio de nuestra Señora, y en un pendón llevasen escripto la vocaçión de su crofadría (sic) de la conçepción, añadido sin pecado original, y consta del grave escandalo que se temía en la ciudad, y para remedio desto el provisor fue en persona al dicho convento, y fulminó processo, en que hubo allegaciones por parte de los dichos frailes y cofadres (sic) y concluso el pleito, les mandó que saliessen con la dicha proçessión como siempre fue uso y costumbre, y el dicho provisor, declaró no ser novedad los letreros de sin pecado original atento que la Iglesia cathólica, y los sacros cánones, y breves de Su Santidad lo permiten, y los dichos frayles apelaron deste mandato, y no quisieron acompañar la dicha cofadría y toda la çiudad que estava a la mira deste caso, se escandalizó notablemente, por saver como se sabía que el no salir los dichos

${ }^{20}$ ACDF, S.O., St. st. M 6 a, f. 6 r.

21 Sul capitolo cattedrale di Cordova cfr. Aranda Doncel, J. 1999. "Los prebendados del cabildo catedralicio de Córdoba durante los siglos XVI y XVII: la provisión de la canonjía magistral", in A.L. Cortés Peña, M. L. López Guadalupe, Estudios sobre Iglesia y sociedad en Andalucía en la edad moderna: 137-152. Granada, Universidad de Granada. 
padres, era porque los dichos cofadres, llevavan los dichos retulos en su cofadría, y porque la dicha ciudad vio que otros religiosos, de otra orden la acompañaron, y de todo lo dicho fueron autores el padre nuestro fray Antonio de Miranda, presidente que era del dicho convento de Regina y fray Francisco del Espíritu Santo y otros padres del dicho convento. ${ }^{22}$

Una ricostruzione di parte, ovviamente, quella offerta a Roma dal prelato sivigliano, ma ciò nondimeno essa risulta altamente significativa. Al fondo si intravede un contrasto che non era semplicemente tra ordini religiosi, e dunque tra tradizioni teologiche diverse e confliggenti. La questione è molto più complessa e sfuggente e si presenta come un conflitto inizialmente tutto andaluso tra capitoli cattedrali e ordinari diocesani (nel caso di Cordova); tra cabildos municipali e nobiltà (bacino d'utenza principale della confraternita in questione e anche di molte altre) ${ }^{23}$ da una parte e ordine domenicano dall'altra; tra vescovi e potere monarchico, tra vescovi e Inquisizione. Se infatti è vero che le effervescenti iniziative immacoliste di Pedro de Castro, il grande protagonista della controversia sulle lamine di Granada ora passato alla pingue sede vescovile sivigliana, e le conseguenti tensioni con il Sant'Uffizio e con Roma possono essere spiegate almeno in parte attraverso il ricorso al consueto prisma del conflitto tra vescovi e inquisitori che aveva caratterizzato la storia spagnola per tutto il Cinquecento, ${ }^{24}$ è altresì vero che, ad esempio, la situazione cordovese si presenta come esattamente opposta e che quindi tale schema esplicativo non può funzionare per tutto il caso andaluso. Tutte queste tensioni sfociarono comunque in una sorta di rivolta generalizzata contro i domenicani: sia nel caso di Siviglia, dove un arcivescovo immacolista attaccava violentemente i domenicani per la difesa pubblica delle loro posizioni, sia a Cordova, diocesi governata invece da un prelato domenicano, il quale doveva affrontare l'opposizione del clero canonicale, che mal sopportava l'anti-immacolismo (e, con ogni probabilità, non solo quello) dell'ordinario.

Siviglia sarebbe comunque diventata il centro focale dello scontro sull'Immacolata negli anni a venire, e la storiografia ha infatti generalmente recepito e interpretato la storia della controversia come una vicenda originata dalle prediche macoliste dei domenicani sivigliani, fomentata da un arcivescovo episcopalista e non molto favorevole ai domenicani come Pedro de Castro, ${ }^{25}$ dai suoi emissari a Roma, il licenciado Bernardo de Toro e l'arcidiacono di Carmona Mateo Vázquez de Leca, e successivamente da un Filippo III desideroso di far sentire direttamente la propria voce a Roma su una questione che si poneva al crocevia tra teologia, politica e devozione popolare. In realtà si trattò di una reazione a catena che fu in grado di incrinare su tutto il territorio della Monarchia precari equilibri all'interno della

${ }^{22}$ Ibidem, f. 77r, miei corsivi, sottolineato nell'originale.

23 Cfr. Sánchez Herrero, J. 1985. Las cofradías de Sevilla: historia, antropología, arte, Sevilla, Universidad de Sevilla.

${ }^{24}$ Così Scaramella, P. 2008: 1008. In generale cfr. Pastore, S. 2003. II Vangelo e la spada. L'Inquisizione di Castiglia e i suoi critici (1460-1598): 349 ss Roma, Edizioni di Storia e Letteratura.

${ }^{25}$ Pedro de Castro si dimostrò semmai vicino alla Compagnia di Gesù sivigliana, visto che conferì agli ignaziani vari compiti di responsabilità all'interno dell'arcidiocesi, come la visita delle scuole, le missioni popolari nelle aree periferiche e maggiormente bisognose di cura spirituale, così come le conferenze morali da impartire al clero secolare. Cfr. Sánchez Herrero, L. 1992. "Sevilla barroca (1581-1700)", in C. Ros, Historia de la Iglesia de Sevilla: 435. Sevilla, Ed. Castillejo. 
compagine ecclesiastica e di sconvolgere i delicati rapporti tra le istituzioni ecclesiastiche e quelle civili. Da Siviglia i disordini si estesero alle province circostanti: un episodio importante ebbe luogo a Toledo nel novembre del 1615, dove i francescani del convento di San Juan de los Reyes annunciarono la difesa di quattro conclusioni teologiche, una delle verteva sulla questione: «Utrum Virgo deipara contraxerit debitum incurrendi peccatum originale», cui venne apposta la postilla «Conclusio est negativa». La reazione dei domenicani di Toledo non si fece attendere: la tesi venne denunciata all'Inquisizione come eretica o semi-eretica. Dopo consultazione di teologi e università il Consiglio della Suprema decretò, nel gennaio del 1616, che la proposizione poteva essere considerata come probabile e quindi pubblicamente difesa. Fu la conferma di ciò che gli immacolisti intimamente già sapevano, ossia che nessun tipo di supporto sarebbe loro venuto dal supremo tribunale della fede spagnolo, organismo in cui l'influenza domenicana era notoriamente preponderante. $\dot{E}$ anche per tale motivo che l'affare arrivò subito a Roma: per il partito immacolista fare appello alla suprema istanza arbitrale in materia di fede voleva dire anche scavalcare le competenze del Sant'Uffizio spagnolo, che certo non aveva il potere di definire un dogma ma avrebbe potuto legittimamente porsi come una prima istanza di giudizio in attesa del pronunciamento pontificio. Paolo $\mathrm{V}$ non ebbe bisogno in questo caso di ricorrere allo strumento dell'avocazione, così come era fragorosamente accaduto sotto Clemente VIII nel 1594 in merito alla disputa de auxiliis.

Negli anni successivi al 1614 le polemiche e i disordini si generarono a tutti i livelli, dalla dotta conclusione teologica nei collegi universitari o negli studia conventuali alla predica nella chiesa principale della città, fino ad arrivare a eventi di ridondante cerimonialità urbana di stampo mariano concepiti proprio allo scopo di polemizzare con il partito macolista; una polemica che aveva destinatari ideali ben precisi e che travalicava talvolta i confini della pratica religiosa e devozionale, fino a trascendere nella messa in scena goliardica e blasfema. È quanto accadde a Siviglia nell'ottobre del 1616, alla vigilia della festività di san Francesco, quando un gruppo di persone diede vita ad una encamisada, ${ }^{26}$ una festa notturna in cui una persona travestita da re di Spagna e un'altra travestita da papa, quest'ultima con uno stendardo recante la scritta «sin pecado original», dopo aver simulato una corrida de toros fecero visita ad una casa di prostitute cantando coplas decisamente sconvenienti. Si trattava di una insolenza così grave che un informatore sivigliano si sentì in dovere di riferire a Roma l'accaduto, precisando anche che, a parte un paio di gentiluomini che avevano animato questi episodi, «todos los demás de la enmascarada eran bodegoneros y mulatos», gente semplice ed illetterata, e che pertanto non sarebbe stato così difficile colpire i pochi - e veri - responsabili dei vergognosi atti. ${ }^{27}$

UN «NEGOTIO GRAVE ET IMPORTANTISSIMO CHE MI HA FATTO PASSARE SENZA SONNO MOLTE E MOLTE NOTTI»: IL NUNZIO PONTIFICIO, SUA MAESTÀ CATTOLICA, L'EPISCOPATO SPAGNOLO

26 II Diccionario de la Real Academia Española del 1732 definisce la encamisada come «cierta fiesta, que se hacía de noche con hachas por la ciudad, en señal de regocijo, yendo a caballo, sin haver hecho prevención de libreas, ni llevar orden de máscara, por haverse dispuesto repentinamente, para no dilatar la demonstración pública y celebración de la felicidad sucedida».

${ }^{27}$ ACDF, S.O., St. St. M 6 a, f. 345r. 
Antonio Caetani era partito alla volta di Madrid nell'ottobre del 1611 forte della pregressa esperienza della nunziatura di Praga, presso la Corte imperiale, carica che disimpegnò tra il 1606 e il $1610 .{ }^{28}$ Formatosi a Roma e poi a Bologna, ove ottenne la laurea in utroque nel 1590, aveva ricevuto un imprinting politicodiplomatico al seguito dello zio, Enrico Caetani, che assistette nella sua missione in Polonia nel 1596 e in occasione della quale fu inviato dapprima presso il duca di Baviera e, al ritorno, presso la Repubblica di Venezia. A Madrid Caetani dovette occuparsi di varie importanti questioni di politica internazionale ma anche di innumerevoli controversie giurisdizionali, di conflitti tra vescovi e capitoli cattedrali (come a Cuenca e nella stessa Siviglia), nonché di censura libraria, specie in occasione delle polemiche suscitate dalla pubblicazione del tomo XI degli Annales ecclesiastici di Cesare Baronio (in cui l'oratoriano prendeva posizione rispetto alla questione della Monarchia Sicula) e della Defensio fidei di Francisco Suárez. ${ }^{29}$ L'eco delle concitate vicende andaluse giunse molto presto a Madrid e Caetani, in qualità di rappresentante ufficiale del pontefice e persona dotata di molteplici prerogative giurisdizionali in materia ecclesiastica, si rese conto subito che si trattava di una questione tutt'altro che marginale.

Alla corte spagnola, da qualche anno trasferitasi a Valladolid, i partiti in conflitto erano tutti presenti e pronti a offrire a Filippo III e ai suoi più stretti consiglieri il proprio personale punto di vista sulla vicenda. II primo resoconto ufficiale del nunzio a Roma è datato luglio 1615, e proprio il 18 di quello stesso mese Caetani aveva emanato un editto che aveva la funzione di riportare la calma nell'infiammata Andalusia. ${ }^{30}$ Ma già agli inizi di settembre i due emissari sivigliani - i già menzionati Toro e Vázquez de Leca - inviarono il loro primo memoriale allo scopo di smentire le voci, che a loro dire i domenicani stavano diffondendo a corte, circa presunte persecuzioni che l'ordine dei predicatori sarebbe stato costretto a subire a Siviglia e di denunciare l'audacia e l'animosità di un ordine religioso che non rinunciava a censurare l'opinione pia come erronea in quanto non conforme alla dottrina di Tommaso. ${ }^{31}$

Si trattava di porre fine ai disordini, alle pubbliche «altercationes», causa di grave scandalo tra i laici, ma non era facile trovare il modo diplomaticamente più adatto per ottenere tale risultato. In gioco non c'era solo la grande suscettibilità di

${ }^{28}$ Cfr. Lutz, G. 1973. "Caetani, Antonio", in Dizionario biografico degli Italiani, vol. XVI: 120125. Roma, Istituto della Enciclopedia Italiana.

${ }^{29}$ Cfr. Borromeo, A. 1982. "Il cardinale Cesare Baronio e la Corona spagnola", in R. De Maio, L. Gulia, A. Mazzacane (eds.), Baronio storico e la Controriforma. Atti del Convegno Internazionale di Studi, Sora 6-10 ottobre 1979: 57-166. Sora, Centro di Studi Sorani «Vincenzo Patriarca»; Lopetegui, L. 1946. "La Secretaría de Estado de Paulo V y la composición del «Defensio Fidei» de Suárez", Gregorianum XXVII: 584-596.

${ }^{30}$ La ricostruzione offerta dalla Relación fatta redigere da Filippo IV accusa il nunzio di aver pedissequamente accolto il punto di vista del domenicano Domingo de Molina, giunto a corte a Madrid da Siviglia proprio nell'estate del 1615 per offrire il punto di vista dei domenicani, visto che nell'editto da lui emanato il 18 luglio si attribuisce tutta la colpa dei disordini agli immacolisti: «Este fray Domingo de Molina, fue el que por el mes de julio de este año diio en el patio de palacio, que Nuestra Señora avía sido concevida como Calvino y Lutero. Con esto de la mano de santo Thomás, y con las afliciones y persecución que persuadió padecía la religión. Movió mucho los ánimos y el nuncio hizo a 18 de julio un edicto para poner freno a estas demasias, cargando en la narrativa a los de la parte pía»; Relación histórico-theológico-política, cit.

${ }^{31}$ BUG, Ms. 13425, ff. 82-84. 
ordini religiosi il cui unico scopo era di rivendicare di fronte a tutto il popolo il proprio maggiore tasso di ortodossia rispetto ai diretti concorrenti: c'era un problema non trascurabile di rispetto di decisioni pontificie pregresse - evidentemente disattese che da un punto di vista politico, ma anche simbolico, mal si accordava con l'emanazione di nuovi provvedimenti in materia (chiesti da più parti, del resto), che avrebbe potuto essere interpretata nelle maniere più varie. E uno degli ostacoli più rilevanti al felice esito della mediazione romana si rivelò ben presto l'atteggiamento dell'arcivescovo Pedro de Castro, individuato da Caetani come il fomentatore della devozione popolare mariana a fini biecamente politici. È questo uno dei nodi centrali della lettura che il nunzio fornisce a Roma delle vicende sivigliane: nelle cose di devozione, di per sé buone e salutari, il popolo va sorvegliato, corretto, contenuto, e non va lasciato andare a briglia sciolta, come stava invece accadendo in Andalusia per iniziativa dell'arcivescovo:

Continuano in Siviglia i medesimi disordini, cagionati particolarmente per rispetto de' religiosi, i quali pare, che convertiscano gli istessi rimedii in danno, nuocendo anche grandemente a questa causa il procedere di quell'arcivescovo, il quale non fa quel che gli tocca, perché appende per cosa di divotione il lasciar correr a redini stese il popolo, dove vuole, e pur in questo caso s'havria a procurar di correggere l'emulatione, e non la devotione, che per sé è santissima: ma col cervello di quel zelante prelato non v'è rimedio, quando inclina ad una cosa. L'istesso disordine s'era suscitato in Madrid, e rimediai facilissimamente, ponendo subito tutte le parti d'accordo. ${ }^{32}$

In un'altra occasione di poco successiva, sempre con riferimento ai moti popolari immacolisti sivigliani, il nunzio faceva sapere che il papa - uso la parafrasi di José Pou y Martí - «reprueba tantas procesiones», che Caetani chiama significativamente «conventículas», notando anche «que los españoles son exagerados en sus usos religiosos y excesivamente crédulos en materia de revelaciones y profecías». ${ }^{33}$ E questa una delle rare occasioni in cui, nella corrispondenza concernente lo scontro sulla definizione dogmatica dell'Immacolata, si fa allusione alle lamine plumbee del Sacromonte di Granada, ma in tema di rivelazioni e profezie si pensi anche a ciò che sarebbe accaduto anni dopo, a controversia già almeno in parte placata, con la pubblicazione dell'opera di suor María de Ágreda, la Mística ciudad de Dios, opera caratterizzata da un robusto contenuto profetico basato proprio sull'Immacolata Concezione della Vergine Maria. $^{34}$

Alla fine di settembre del 1615 Caetani tornava ad accusare pesantemente l'arcivescovo Castro, che invece di svolgere il suo ruolo naturale di mediatore, «s'ha guadagnato l'opinion di parte, c'ha difficoltato tanto più il rimedio». Nel giugno

\footnotetext{
${ }^{32}$ Antonio Caetani al cardinale Borghese, 28 settembre 1615, Ibidem, ff. 22rv.

${ }_{33}$ Pou y Martí, J.M. 1931. "Embajadas de Felipe III a Roma pidiendo la definición del dogma de la I. Concepción de María", Archivo ibero-americano XVIII, 34: 381.

${ }^{34}$ Cfr. Mendia, B., Artola Arbiza, A.M. 2005. La ven. m. María de Jesús de Ágreda y la Immaculada Concepción: el proceso eclesiástico a la «Mística ciudad de Dios», Ágreda, Concepcionistas Franciscanas; Cabibbo, S. 2003. "Una profetessa alla corte di Spagna. II caso di Maria d'Agreda fra Sei e Settecento", Dimensioni e problemi della ricerca storica 1: 83-105; Ead. 2006, "Vizi e virtù di una «sociedad ensimismada». Maria d'Agreda e la Spagna di Filippo IV", Península. Revista de estudios ibéricos 3: 165-172; Ead. di prossima pubblicazione. Maria d'Agreda e l'affaire della Mystica ciudad de Dios, Roma, Viella.
} 
dell'anno successivo il nunzio se la sarebbe presa con i «processi» intentati dall'arcivescovo per «parole impertinenti dette da' domenicani ne' pulpiti», ma aggiungendo: «delle quali Dio sa se egli stesso n'habbia havuto più colpa, che i medesimi Padri per la disperatione nella quale gli ha posti». ${ }^{35}$ Caetani non cedeva dunque alla tentazione di dare credito alla versione dominante (che era anche quella di Castro) secondo la quale gli scontri erano stati causati dall'insolenza dei domenicani. Tra i due partiti - afferma Caetani - forse è proprio quello immacolista a non essersi contenuto all'interno dei limiti prescritti dalle costituzioni di Sisto IV e di Pio V, ma realisticamente nota anche che «saria nondimeno stata più prudenza d'essi domenicani cedere, e tacere, già che hanno tutto il popolo contrario». ${ }^{36}$

Analisi sottile della vicenda in atto quella fornita da Caetani alla corte di Roma, che si fa specchio di una strategia volta a usare la massima prudenza in una questione che non era possibile circoscrivere facilmente al solo ambito degli affari ecclesiastici. È lo "scandalo" a preoccupare il nunzio, ossia il coinvolgimento dei laici, un coinvolgimento a volte attivo, a volte passivo, ma sempre disdicevole e pericoloso: «Gran parte del male consiste ne' laici medesimi», ebbe a sottolineare nel febbraio del 1616. ${ }^{37}$ Esattamente per questo motivo bisognava, secondo Caetani, evitare di ricorrere a un pubblico precetto che imponesse ai domenicani il silenzio nelle dispute e nelle prediche in materia di Immacolata Concezione, così come i loro avversari chiedevano. $E$ in tale direzione il nunzio si mosse con solerzia ma anche grande circospezione, così come illustra in una lettera indirizzata al cardinale Borghese:

Li domenicani da alcun tempo in quà hanno lasciato di parlare etiam dentro $\mathrm{i}$ termini, che non sariano stati impediti dalle bolle, perché io secretamente hò loro imposto così, già che ogni loro detto in tal materia in cospetto del popolo suona male, e ne risulta pregiudicio commune e loro particolare ancora, ma più oltre di quello che contengono i motuproprii, non ho voluto gravarli per ordine pubblico, per non far loro affronto, e metterli in confusione e disperatione con trionfo degli altri; che questo mi saria parso non manco contra le regole del buon governo, che contra quelle della charità. Dice però il vero monsignor arcivescovo che la seditione nel publico sta più sedata, e la causa n'è quello, ch'io dico; ma tale, che nell'intrinseco, e secretamente va tanto più serpendo, et apostemandosi, quanto detto monsignore di natura litigioso s'è reso sospettissimo alli domenicani, e dichiarato capo dell'altra parte, perché sotto pretesto della devotione tiene qua huomini destinati, che prima fecero instanza a Sua Maestà, che rinnovasse un'ordine in parte simile a quello del re don Giovanni I d'Aragona sopra questa materia, e poi non riuscendoli questo, che almeno si facesse una giunta di prelati, acciò si supplicasse Nostro Signore a ponervi le mani, e farvi su qualche nuova dichiaratione in destruttione dell'altra opinione. $^{38}$

\footnotetext{
${ }^{35}$ Antonio Caetani al cardinale Giangarzia Millini, 16 giugno 1616, ACDF, S.O., St. st. M 6 a, f. $261 r$.

${ }^{36}$ Lettera del 31 luglio 1615, Ibidem, f. 10r.

${ }^{37}$ Antonio Caetani al cardinale Varallo, 24 febbraio 1616, Ibidem, f. 93r.

${ }^{38}$ Antonio Caetani al cardinale Borghese, 24 marzo 1616, Ibidem, ff. 95v-96r (miei corsivi).
} 
Ed ecco che una questione teologica, con importanti risvolti sul piano devozionale, si stava velocemente trasformando, sotto gli occhi del nunzio Caetani, in una questione politica a tutto tondo. Castro aveva chiesto a un padre gesuita, Juan de Pineda, di comporre un opuscolo - per di più in volgare - in favore della Pragmática del 1394 di re Giovanni I d'Aragona (detto il Cazador), che ordinava ai suoi sudditi di celebrare in maniera solenne la festività dell'Immacolata Concezione proibendo allo stesso tempo ai religiosi, sotto gravi pene (tra cui l'espulsione dal Regno), di predicare l'opinione contraria a quella pia. ${ }^{39}$ Si trattava evidentemente di un precedente lontano la cui rievocazione non poteva che irritare la Santa Sede: un principe secolare, tra l'altro da molti considerato scismatico in quanto nel novero di coloro che avevano riconosciuto come legittimo l'antipapa Clemente VII, che si era spinto così in là nella sua autonomia dalla Chiesa di Roma da impartire disposizioni (e comminare pene) su una questione di natura strettamente dottrinale come quella del concepimento senza macchia della Vergine, veniva ora presentato da un gesuita come la scaturigine di una legittima consuetudine dei regni appartenenti alla Monarchia spagnola, tanto più necessaria quanto più si faceva attendere il definitivo pronunciamento della Santa Sede in materia. II nunzio esprimeva nel memoriale le proprie riserve circa l'opportunità di una totale imposizione del silenzio ai macolisti, in quanto ciò avrebbe dato adito a «murmuraciones» da parte degli eretici circa la sentenza affermativa: se si impone il silenzio vuol dire che è sbagliata, ma se è sbagliata perché non la si condanna ufficialmente? E se invece è giusta, perché si proibisce di predicarla ${ }^{40}$ Caetani si rendeva perfettamente conto di tutti i rischi di una tale rievocazione e nella sua lettera al nipote di Paolo $\mathrm{V}$ attaccava violentemente la pretesa delle autorità spagnole di ritenersi indipendenti da Roma in questioni squisitamente ecclesiastiche e dottrinali, una pretesa foriera di uno scisma non dissimile da quello provocato da Enrico VIII d'Inghilterra quasi un secolo prima:

Se si permette non solo che si disprezzino così i motuproprii, il che al fine è il minore inconveniente di tutti in questo caso, ma che anco in poca stima delle chiare determinationi della santa Chiesa seguite appresso s'habbiano a commentare come testi sacrati de determinationi de' re laici in cosa pura ecclesiastica nulle per se stesse et invalide, e di già antique et obsolete, et havendo fonti donde toglier l'acqua pura si dia al popolo che le toglia da lagune così sordide e putrefatte, questo non è altro che dar adito un giorno ad un re di Spagna, che senza avedersene possa diventar scismatico, anzi di più cader à poco à poco con tutti i suoi vassalli nella abominevol heresia del regno d'Inghilterra. ${ }^{41}$

Era la suscettibilità di Roma sulla questione della propria autorità esclusiva di determinare in materia di fede il punto sensibile che entrambe le parti cercavano di

${ }^{39}$ Declaración y advertencias del p. Juan de Pineda acerca de la fiesta y celebridad de la Concepción de la Virgen, s.I. e s.a. Cfr. Sommervogel, C. 1895. Bibliothèque de la Compagnie de Jésus: vol. VI, cc. 796-801. Bruxelles-Paris, O. Schepens-A. Picard. Varie furono per l'occasione le stampe che riproducevano il testo della Pragmática di Giovanni I d'Aragona, con tanto di traduzione in castigliano a fronte. Una è ad esempio consultabile in ACDF, S.O. St. St. M 6 a, ff. 167r-168v (De los privilegios del sereníssimo señor don Juan, de gloriosa memoria, rey de Aragón, hiio y sucessor del señor rey don Pedro). Su Juan de Pineda, che ebbe un ruolo di primo piano nella storia della censura libraria del XVII secolo in Spagna, si veda Olivares, E. 1988. "Juan de Pineda (1557-1637). Biografía. Escritos. Bibliografía", Archivo Teológico Granadino LI: 5-133.

${ }^{40}$ Cfr. Pou Y Martí, J.M. 1931: 382.

${ }^{41}$ ACDF, S.O., St. st., M 6 a, ff. 96v-97r. 
stuzzicare per volgere la situazione a proprio vantaggio. Come ad esempio quando, nel dicembre del 1615, il domenicano Gabriel Vázquez denunciò al Sant'Uffizio romano l'agostiniano spagnolo Cipriano de Pineda, reo di aver affermato pubblicamente: «Quando en estas cosas el pueblo cristiano comúnmente y los hombres doctos dicen una cosa, no es menester aguardar al Pontífice para tenerlo por cierto y por de fe». ${ }^{42}$ La situazione si presentava come di difficile gestione; quanto al libro di Pineda Caetani fece presenti le proprie perplessità al cardinale Borghese: «Se fusse officio del mio Tribunale prohibire questo libro, l'havrei subito fatto, ma questo per esser insolito sarebbe stato inteso male, e forse ne meno ubbidito»; ma d'altronde anche il coinvolgimento dell'Inquisizione spagnola nella questione presentava non pochi inconvenienti agli occhi del nunzio.

Nel luglio del 1615 il cardinale di Toledo e inquisitore generale, Bernardo de Sandoval y Rojas, aveva scritto ai cardinali della congregazione romana del Sant'Uffizio per metterli al corrente della misura da lui presa per i territori spagnoli che imponeva, sotto gravi pene, la stretta osservanza delle costituzioni apostoliche e dei decreti tridentini in materia di Immacolata e vietava per il momento le discussioni, sia pubbliche che private, sulla stessa questione. II 28 settembre lo stesso Inquisitore generale chiedeva a Roma di emettere un breve mediante il quale «en las cátedras y en escuelas, y libros de latín, se pueda disputar y defender la opinión de que non fuit concepta in pecato originali, pero que fuera de esto en los púlpitos, ni en ninguna otra parte, no se muestre la opinión contraria a la dicha, porque dello resultaría un escándalo y desconsuelo increyble en todo el mundo». ${ }^{43}$ Roma dal canto suo rispondeva che, dal momento che i due partiti in lotta non si astenevano dagli scontri pubblici nonostante gli ordini dati dal nunzio, il Sant'Ufficio chiedeva ufficialmente all'Inquisitore generale di interporre «l'autorità sua, et di cotesta Inquisitione, acciò almeno in questo modo venga raffrenato l'ardire delli temerari et disubidienti delle constitutioni apostoliche, decreti del sacro concilio Tridentino, et delli ordini del nuntio di questa Santa Sede apostolica». ${ }^{44}$

Questo intervento ufficiale del supremo tribunale della fede spagnolo per punire la mancata osservanza dei brevi pontifici era tuttavia sconsigliato dal nunzio Caetani in virtù della sua conoscenza della situazione politica spagnola e anche della mentalità dei cristianos viejos sudditi di Sua Maestà cattolica per ciò che attiene all'azione inquisitoriale. Nel settembre del 1616 scriveva infatti al cardinale Millini che per «aggratiarse anche la nobiltà» i prelati spagnoli che si erano messi a capo del movimento immacolista (e Castro prima di tutto) avevano diffuso la voce che nella bolla in preparazione a Roma sarebbe stata contenuta la clausola secondo la quale «il Santo Officio possa procedere contra i contraventori»; ciò era secondo Caetani alquanto pericoloso, perché il solo nome "Sant'Uffizio" - si diceva in Spagna - «è cosa horribile, e la semplice inquisitione dà nota, et infama le famiglie, sonarebbe in Spagna male se mai alcun venisse processato per haver diffeso la limpiezza della pura Concettione». ${ }^{45}$ Per lo stesso motivo anche l'emanazione di un provvedimento pontificio, aggiuntivo rispetto a quelli passati di Sisto IV e di Pio V, sarebbe stato interpretato in Spagna in maniera errata e

\footnotetext{
${ }^{42}$ Ibidem, f. 31r.

43 Ibidem, M 6 a, f. 23r.

44 Ibidem, M 6 a, f. 17v.

45 Ibidem, M 6 a, f. 271 r.
} 
tendenziosa. Si trattava di una questione di forma, non di sostanza, da una parte a causa delle «spropositate intelligenze, che danno alle parole delle Bolle ciascuno a modo suo», ${ }^{46}$ dall'altra perché la materia si era talmente trivializzata, era divenuta talmente popolare, che sarebbe bastato a uno di questi "prelati sobillatori" diffondere la voce che il motuproprio era poco favorevole alla causa immacolista per generare l'ennesima insurrezione. Le parole e le azioni andavano soppesate al massimo grado. Per il nunzio la ricetta vincente era la seguente: in assenza di un'effettiva collaborazione da parte delle autorità ecclesiastiche e secolari spagnole per pacificare gli animi, «solamente con la rinovatione dei motuproprii, e con dar di secreto ordine ai domenicani, che tacciano la cosa saria sopita». ${ }^{47}$

Antonio Caetani si trovò a dover gestire una mediazione difficile, per di più in un clima politico-religioso surriscaldato dall'esplosione di una straripante e polemica devozione popolare immacolista, da prelati che non esitavano ad approfittarne, istigando e indirizzando tale devozione, che non provvedevano a correggere l'ignoranza del volgo in materie delicate da un punto di vista teologico, che commissionavano persino la redazione di libelli gravemente lesivi delle prerogative della Santa Sede, e anche da autorità politiche che invece di intervenire prontamente per quietare gli animi, lasciavano correre, come ebbe a lamentarsi lo stesso Caetani. Nella sua lettera al cardinal Borghese del 24 marzo del 1616 il nunzio offriva un ritratto per certi versi assai sconsolato della nazione spagnola, devotissima alla Chiesa ma anche, proprio per questo motivo, troppo incline a esagerazioni devozionalistiche, potenzialmente molto pericolose per il corretto rapporto che avrebbe dovuto instaurarsi tra sfera ecclesiastica e sfera civile:

Qui si tiene una opinione, che non corretta non è admittibile, ciò è che al popolo in tutto e per tutto s'habbia d'assecondare in materie simiglianti, per non dargli occasione di sollevarsi. Ben è vero, che si ha da assecondare in tutto quello che è devotione, ma ciò si ha da intendere con illuminarlo, e levarlo insieme da ignoranza, e non lasciarlo correre trabocchevolmente alla cieca dove vuole, perché questo non è altro, che farlo assoluto arbitro e giudice di quello che à lui non tocca; onde facilmente corra poi a precipitarsi. La natione spagnola è devotissima, ma l'istessa pietà opera, che in simili materie, come anche in santificatione e beatificatione de' santi, et in tutto quello, che ha del miracoloso e del pio trascorre con manco consideratione di quello che si converria; e però come a' prelati tocca aiutar la pietà quando va sicura per buon camino, così illuminarla dove si veda errar per le tenebre, e che potria pericolare, di sorte che le pecore odano, e seguano la voce del pastore, e non il pastore quella delle pecore. ${ }^{48}$

Prudenza, discrezione, circospezione: queste le parole d'ordine dell'arcivescovo di Capua per la gestione della controversia sull'Immacolata Concezione. Una cautela che lo spinse perfino a sconsigliare di dare eccessiva pubblicità - in Spagna come altrove - all'emanazione del breve del 6 agosto 1616, con il quale Roma ribadiva il dovere di obbedienza alle costituzioni vigenti in materia e vietava ai contendenti di condannare come eretica l'opinione contraria; questo perché bisognava evitare qualsiasi occasione di scontro di piazza, e a tal fine la comunicazione del breve sarebbe dovuta avvenire non «per le strade, o nel pulpito»

\footnotetext{
${ }^{46}$ Antonio Caetani al cardinale Giangarzia Millini, 22 maggio 1616, Ibidem, f. 213r.

${ }^{47}$ Antonio Caetani al cardinale Giangarzia Millini, 15 settembre 1616, Ibidem, f. 271 r.

${ }^{48}$ Antonio Caetani al cardinale Borghese, 24 marzo 1616, Ibidem, ff. 98v-99r (mio corsivo).
} 
ma «ai capi [degli ordini] e con la circospettione e cautela, che lo sopradetto». ${ }^{49}$ Stesse identiche accortezze la Congregazione del Sant'Ufficio impose del resto nei territori italiani soggetti alla sua giurisdizione, visto che sin dai giorni immediatamente successivi all'emanazione del breve gli inquisitori ricevettero precisa disposizione di non darne pubblicità al di fuori dei conventi degli ordini religiosi, e soprattutto di raccogliere le denunce circa gli eventuali trasgressori senza però procedere direttamente. II 18 agosto l'inquisitore di Genova, Eliseo Masini, confermava al cardinal Millini di aver ricevuto l'ordine «intorno al procedere con quelli, che non osservaranno la nuova costitutione innovatoria di quelli di Sisto quarto, e di Pio $\mathrm{V}$ sopra la concettione della santissima Vergine, con pigliar le denoncie, ch'in tal caso mi saranno date, et mandarle a cotesta sacra et suprema Congregatione». ${ }^{50}$ Similmente l'inquisitore di Venezia scriveva allo stesso cardinale di aver compreso che «contro i trasgressori possino procedere non solo gli Ordinarii, ma anco gl'Inquisitori accettando però le denuncie, et poi dando aviso a cotesta Sacra Congregatione, et aspettando risposta di quanto si dovrà fare». ${ }^{51}$

Quanto alla definizione dogmatica dell'Immacolata, una richiesta che arrivò a Roma, in maniera si potrebbe dire informale, già dai primi momenti dello scontro e nelle prime comunicazioni da parte delle autorità ecclesiastiche e civili andaluse, Caetani spiegava all'arcivescovo di Burgos, Fernando de Acevedo, membro del Consejo de Castilla, che non c'erano le condizioni per compiere un tale passo. ${ }^{52}$ Per Roma gli elementi offerti dalla Sacra Scrittura e dai Padri della Chiesa non erano sufficienti per un pronunciamento ex cathedra del pontefice (con la sola assistenza dello Spirito Santo); ${ }^{53}$ la prudenza (anche in considerazione dell'immagine che si voleva trasmettere della Chiesa cattolica agli eretici) imponeva dei modi politicamente condivisi di definire una questione teologica controversa, e tra questi il più adatto sarebbe stato la convocazione di un concilio. Si tratta di un argomento importante, che sarebbe tornato più volte - e utilizzato in maniere diametralmente opposte - nei tesi rapporti tra la Santa Sede e la Monarchia spagnola negli anni a venire.

\section{DIPLOMAZIA TEOLOGICA: INVADENZA ASBURGICA E CAUTELE ROMANE}

Non è semplice individuare con precisione il momento in cui Filippo III iniziò a interessarsi direttamente alla questione dell'Immacolata e a considerarla una questione politica importante, così importante da creare una Real Junta de la Inmaculada Concepción e persino inviare a Roma degli emissari con il compito di fare pressioni sul pontefice per giungere alla definizione dogmatica. Dalla corrispondenza indirizzata ai membri della Congregazione romana del Sant'Ufficio traspare un atteggiamento di iniziale prudenza e rispetto delle prerogative pontificie

\footnotetext{
${ }^{49}$ Antonio Caetani all'arcivescovo di Burgos, 4 settembre 1616, Ibidem , f. $273 \mathrm{v}$.

${ }^{50}$ L'inquisitore di Genova al cardinale Giangarzia Millini, 18 agosto 1616, Ibidem, f. 314r.

${ }^{51}$ L'inquisitore di Venezia al cardinale Giangarzia Millini, 6 agosto 1616, Ibidem, f. 310r.

${ }^{52}$ Antonio Caetani all'arcivescovo di Burgos, 4 settembre 1616, Ibidem, M 6 a, f. $274 \mathrm{v}$.

${ }^{53} \mathrm{Il}$ concetto di assistenza dello Spirito Santo è centrale nei dibattiti sull'infallibilità papale che culminarono nella costituzione Pastor aeternus del 18 luglio 1870 adottata dal concilio Vaticano I.
} 
da parte del sovrano asburgico e dei suoi più diretti collaboratori. Nell'aprile del 1616, per esempio, il nunzio Caetani riportava al cardinale Borghese una conversazione da lui avuta con il duca di Lerma e la dura reazione di quest'ultimo rispetto all'iniziativa del viceré di Maiorca di pubblicare, in occasione della Settimana Santa, l'editto di Giovanni I d'Aragona; il Valido, scrive Caetani, «apprese l'attione per malissima, e soggiunse, che se Sua Maestà si governarà per suo consiglio, questo fatto non sarebbe restato impunito». ${ }^{54}$ Stessa opinione negativa Lerma avrebbe avuto - stando alla testimonianza di Caetani - del libello di Pineda. Da una parte c'è quindi la diplomazia pontificia che si adoperava per una veloce tacitazione dei disordini generati dagli eccessi popolari immacolisti, dall'altra una corte asburgica che, nel quadro di una iniziale cautela nei suoi rapporti con il nunzio pontificio, nel migliore dei casi tollerava tali eccessi, e che si sarebbe presto convertita alla causa della richiesta della definizione dogmatica.

Nell'estate del 1616 l'intenzione di inviare a Roma un rappresentante diplomatico straordinario era già nell'aria, tanto che l'emanazione del breve del 6 agosto venne vista anche come un modo per impedire la concretizzazione di tale progetto. Tutto si giocò all'interno di una Junta de Prelados che Caetani aveva accettato di presiedere (e le cui riunioni si svolsero all'interno della sua abitazione) ${ }^{55}$ ma la cui creazione era stata insistentemente richiesta dai due canonici sivigliani, molto attivi a corte in quel periodo, in nome e per conto dell'arcivescovo di Siviglia Pedro de Castro. Compito di questa Giunta sarebbe stato quello di analizzare la documentazione a disposizione (lettere, memoriali, testimonianze), la maggior parte della quale prodotta dai personaggi poc'anzi citati, per decidere una linea di azione finalizzata alla cessazione degli scandali. Un duro colpo, quindi, per le prerogative del Sant'Uffizio spagnolo, ancora una volta scavalcato nelle sue competenze («il tribunale dell'Inquisitione non è stato mai stimato quà molto à proposito per essecutione di quelli rimedii», ${ }^{56}$ scriveva Caetani). Quasi a voler giustificare agli occhi del pontefice, e della Congregazione del Sant'Ufficio, il proprio coinvolgimento nella formazione di un organismo investito di una questione di carattere dottrinale su cui poteva ricadere il sospetto di essere dominato dal sovrano, Caetani si sforza nelle sue lettere di mettere in evidenza l'inefficienza e l'inutilità della Junta. II nunzio scrive infatti nel luglio del 1616 che essa, composta inizialmente dall'arcivescovo di Santiago de Compostela, Juan Beltrán de Guevara, dal vescovo di Cuenca, Andrés Pacheco, e dal vescovo eletto di Valladolid, Francisco Sobrino, era stata costituita sapendo il sovrano «questa materia essere propria di Nostro Signore e non altri», e che inoltre non si era riunita molte volte e che non era giunta a risultati concreti degni di nota. ${ }^{57}$

Nel mese di agosto, in concomitanza con la pubblicazione del breve pontificio, Caetani si adoperò da una parte per chiudere definitivamente e ufficialmente la Junta, dall'altra per recarsi personalmente a corte al fine di trattare la questione e impedire con ogni mezzo l'invio a Roma dell'ambasciatore straordinario di Sua Maestà, già individuato nel frate benedettino Plácido de Tosantos, predicatore ed ex provinciale spagnolo del suo ordine. L'elemento degno di nota è che, secondo il

\footnotetext{
${ }^{54}$ Antonio Caetani al cardinale Borghese, 3 aprile 1616, ACDF, S.O., St. st., M 6 a, f. 94 r.

55 Secondo lo storico Meseguer Fernández la Giunta venne invece presieduta dall'arcivescovo di Burgos, Fernando de Azevedo, presidente del Consejo de Castilla.

${ }^{56}$ Antonio Caetani al cardinale Giangarzia Millini, 16 giugno 1616, ACDF, S.O., St. st., M 6 a, f. $259 \mathrm{v}$.

57 «Detta giunta s'è fatta rarissime volte, et in quelle non s'è concluso niente», scriveva Caetani a Millini già nel luglio 1616, Ibidem, f. 264r.
} 
nunzio, tale progetto di invio di un emissario a Roma era stato concepito proprio in seno alla Junta e, ovviamente, contro il suo volere: «l prelati della Giunta prima di finirla hanno fatto tanto con Sua Maestà che di nascosto impetrarono che si segnalasse persona per andare a Roma che è un certo padre Tosantos Benedettino». ${ }^{58} \mathrm{E}$ non solo: è qui che l'atteggiamento di Filippo III inizia a cambiare, se è vero che per bocca dell'arcivescovo di Burgos il sovrano fece sapere al nunzio Caetani che avrebbe preferito che il breve fosse stato pubblicato solo dopo l'arrivo a Roma del suo emissario, munito dei pareri della Junta, per fare in modo che il pontefice avesse potuto decidere sulla questione con maggiore cognizione di causa. La reazione di Caetani è immediata e stizzita, intravedendo in questa presa di posizione un pretesto per non eseguire quanto ordinato nel breve e anche l'ombra delle manovre a corte dei canonici sivigliani:

Io di questa ambasciata m'alterai molto immaginandomi, come era certo, che questa fusse stata negotiatione di questi canonici di Siviglia con l'appoggio de' prelati, che gli favoriscono, rappresentando a Sua Maestà qualche cosa à lor modo, et in particolare che se ne saria potuto alterar il populo, e così senza perder tempo fui subito dal presidente a dolermi di tal ambasciata, et a rappresentar ragioni invittissime per le quali non si doveva né si poteva di nessun modo impedir tal'essecutione respondendo alle inventioni de' canonici, e sciogliendo tutti gli obietti in contrario. ${ }^{59}$

Oscure rimangono però le ragioni di tale inversione di rotta di Filippo III: è possibile presumere che il sovrano asburgico sia potuto arrivare a decidere di far sua la causa della definizione dogmatica dell'Immacolata Concezione solo a seguito delle abili pressioni dei canonici sivigliani, dell'arcivescovo Castro oppure delle devote insistenze della zia, suor Margherita de la Cruz? ${ }^{60}$ Può per esempio aver avuto un ruolo il fatto che sono questi gli anni decisivi del declino del potere del duca di Lerma, preludio all'ascesa del duca di Uceda, al processo che Lerma dovette in seguito subire e alla nomina di Luis de Aliaga, uno degli artefici della sua caduta in disgrazia, a Inquisitore Generale nel $1619 ?^{61}$ La corrispondenza del nunzio ci mostra, almeno fino agli inizi del 1616, un Filippo III apparentemente rispettoso delle prerogative della Santa Sede e del tentativo di mediazione messo in atto da Caetani. ${ }^{62}$ E tra la primavera e l'estate del 1616 che le cose cominciano a cambiare, e il resoconto dello stesso nunzio è fortemente indicativo della percezione

${ }^{58}$ Antonio Caetani alla Congregazione del Sant'Uffizio, 13 agosto 1616, Ibidem, f. 266r.

${ }^{59}$ Antonio Caetani al cardinale Giangarzia Millini, 15 settembre 1616, Ibidem, f. 270 r.

${ }^{60}$ È quanto lascia intendere la maggior parte della storiografia sull'argomento: si veda ad esempio Cortés Peña, A.L. 2001. "Andalucía y la Inmaculada Concepción en el siglo XVII", in Id. (ed.), Religión y política durante el Antiguo Régimen: 103-148. Granada, Universidad de Granada.

${ }^{61}$ Cfr. Feros, A. 2002. El duque de Lerma. Realeza y privanza en la España de Felipe III. Madrid, Marcial Pons; Williams, P. 2008. "El favorito del rey: Francisco Gómez de Sandoval y Rojas, $\checkmark$ marqués de Denia y I duque de Lerma", in J. Martínez Millán, M.A. Visceglia (eds.), La Monarquía de Felipe III: la Corte, vol. III: 185-259. Madrid, MAPFRE.

${ }^{62}$ «E perché gran parte del male consiste ne' laici medesimi, ho di più caminato di conserto con Sua Maestà, la quale si dignò ultimamente scriverne al Magistrato secolare ordinando, che in questo non s'uscisse un punto da far quello, che da me fusse commandato, che s'esseguisse», Antonio Caetani al cardinale Varallo, 24 febbraio 1616, ACDF, S.O., St. st. M 6 a, f. 93v. 
della delicatezza della posta in gioco, potenzialmente problematica persino per la posizione del nunzio nei confronti del pontefice:

I due canonici di Siviglia, che furono già mandati da quell'arcivescovo al re fin da quando Sua Maestà stava in Vagliadolid, havendo continuato per dieci mesi di dar memoriali alla Maestà Sua in nome di esso arcivescovo acciò si fusse fatta una giunta di prelati, dove si fusse trattato di questa materia direttivamente però ad essaminare le cause di supplicar Nostro Signore per la decisione e terminatione per sempre di questa questione, et havendo frà tanto la maestà sua più volte ributtatili, et in specie a Burgos fatto loro intendere per mio mezzo, che saria stato bene, che se ne fussero andati, ne havendo per questo gl'istessi mai desistito, anzi implorato da più parti i gravissimi mezzi e di vescovi, e d'altri per questo loro intento, finalmente parendo a Sua Maestà, che tuttavia la conturbatione in questi suoi regni per la sopradetta controversia andasse continuando, e che fusse necessario darvi qualche ordine, e forma, perché il male non serpesse più avanti [...] pigliò risolutione, per mezzo dell'arcivescovo di Burgos farmi sapere, che conoscendo Sua Maestà, questa materia esser propria di Nostro Signore e non altri, le sarebbe stato caro, che si fusse fatta una giunta, della quale io fussi stato il capo, dove si vedessero le scritture che detti canonici havevano date, e la pretensione d'esso arcivescovo di Siviglia, et insieme si considerasse, che rimedio si fusse potuto dare alli disordini imminenti, e di che così si fusse potuto supplicare la Santità Sua.

L'immagine che si vuole dare a Roma è quella di un riluttante Filippo III portato a cedere di fronte alle asfissianti richieste dei canonici sivigliani. Ma quegli stessi canonici nell'ottobre del 1616 sarebbero stati inviati dal sovrano nell'Urbe con una funzione di supporto rispetto agli ambasciatori straordinari; si tratta dunque di una spiegazione che non può reggere di fronte a ciò che la questione dell'Immacolata sarebbe diventata di lì a pochi anni e anche alla luce della gravità delle interferenze che Filippo III aveva già esercitato nella controversia de auxiliis. Rispetto alle iniziali cautele l'impressione è che il sovrano asburgico si renda presto conto dell'importanza politica dell'affare e che per tale motivo assuma un atteggiamento più che determinato. La questione dell'Immacolata cominciò a perdere i connotati di un affare tutto andaluso che approdava a Valladolid e successivamente a Roma, per assumere sempre di più quello di uno scontro dottrinale a tutto tondo che vedeva come protagoniste le maggiori famiglie religiose e nell'ambito del quale il Papato e la Monarchia cattolica misuravano le proprie forze nell'affermazione l'una della sua esclusiva competenza e sovranità nel giudizio delle controversie di fede, l'altra del proprio ruolo, unico nel mondo cattolico e storicamente giustificato, di difensore e persino di guida della Chiesa.

In Spagna venne sempre più emergendo la rivendicazione di un certo grado di autonomia rispetto a Roma su questioni di dottrina, per la cui definizione si affermava che fosse sufficiente il comune sentire dei fedeli supportato dall'evidenza dell'antichità e della purezza del cattolicesimo spagnolo. In un memoriale inviato il 16 giugno 1617 da Siviglia a Roma e indirizzato a Bernardo de Toro forte è ad esempio l'espressione dello stupore rispetto alla prudenza - vicina in verità alla ritrosia e alla franca diffidenza - che Roma mostrava nei confronti della tanto sospirata definizione dogmatica. La questione - afferma il redattore del memoriale, Pedro de Hojeda - in Spagna è così lampante che davvero non si capisce come può Roma avere ancora dei dubbi: se la Chiesa ha istituito la festa dell'Immacolata, 
questo è già di per sé prova del fatto che si tratta di una verità di fede, e se san Tommaso non aveva dichiarato l'Immacolata verità di fede è solo perché a quel tempo la festa non era stata ancora istituita. Non è possibile poi - prosegue Hojeda - che una festa possa essere istituita per celebrare entrambe le opinioni; e qui il redattore se la prende in particolare con Roberto Bellarmino il quale, controcorrente rispetto al proprio ordine, aveva sostenuto esattamente questa posizione, dichiarando che con la festività dell'8 dicembre la Chiesa non aveva inteso celebrare il concepimento immacolato di Maria bensì semplicemente il suo concepimento. ${ }^{63}$ «Por donde - continua il memoriale - echo de ver quan lubico es esso por allá [Roma], siendo acá [in Spagna] y deviendo ser donde quiera la cosa más clara del mundo». ${ }^{64} \mathrm{~A}$ essere interessato era dunque anche il delicato campo della liturgia, visto che in gioco c'era il senso da attribuire a una festività inserita nel Breviario Romano. ${ }^{65}$

Lo scontro tra ordini religiosi su una materia di carattere dottrinale, a pochi anni di distanza dalla de auxiliis, offriva a Filippo III - e in maniera amplificata, in virtù delle particolari implicazioni ideologiche dell'Immacolata - una nuova occasione per contestare a Roma la sua naturale funzione mediatoria nelle controversie dottrinali sotto il pretesto della necessità della definizione dell'ortodossia religiosa; tutto ciò in nome dell'assolvimento del compito primario della Monarchia spagnola: la difesa della Chiesa. L'ideologia politica degli Asburgo di Spagna aveva bisogno di una ortodossia, da difendere e propagandare, ma la definizione di tale ortodossia sfuggiva al suo controllo. È per tale ragione che la Spagna rivendicava uno specifico diritto di intervento e di interferenza sulle questioni di dottrina in quanto corollario della pienezza delle prerogative della Monarchia in ambito politico e in virtù dei meriti specifici che nella storia la Spagna aveva accumulato al servizio della difesa della fede. Un programma politicoreligioso la cui costruzione subì un'accelerazione proprio agli inizi del XVII secolo, in corrispondenza con il definitivo tramonto dei sogni di supremazia continentale coltivati da Carlo V, i cui echi erano stati ancora presenti nel corso del lungo regno di Filippo II.

63 «Fundamentum huius festi precipuum non esse Conceptionem immaculatam, sed simpliciter conceptionem matris Dei. Qualiscumque enim fuerit illa conceptio, eo ipso, quod conceptio fuit matris Dei, singulare gaudium affert mundo eius memoria; tunc enim primum habuimus pignus certum redemptoris», Bellarmino, R. 1857. Opera omnia: De Ecclesia triumphante, sive de gloria et cultu sanctorum: II-555 (libro III, cap. XVI). Napoli, apud Josephum Giuliano editorem.

${ }^{64}$ BUG, Ms. 13425, f. 225r.

65 In una lettera del francescano Francisco de San Joseph Suessa è proprio questo punto ad essere messo al centro della polemica con i domenicani: «Pero no puedo dejar de dolerme, de que si la Iglesia declara, celebrar ella la Concepción en el sentido de nuestra opinión, dejando todavía libertad de sentir la contraria, destruimos quanto los oppuestos y nosotros avemos dicho y escrito de la zertidumbre y verdad que insinua el hazer la Iglesia fiesta a un Misterio, y lo que diio san Thomás después de san Agustín y san Ildephonso de la de la (sic) Natividad. Pues los dominicos se nos confiessan en sus escritos por vencidos si la Iglesia celebrasse en nuestro sentido: y por la fuerza que esto tiene lo an negado y niegan tenacísimamente. [...] Y no se, si estuviera mejor sacar solamente declaración de que la lglesia celebra en el sentido de preservación. Pues mucho más nos dan ellos y confiessan, si deste sentido de la Iglesia constasse. Y por sus escritos mismos les hiciéramos guerra para necessitarlos a dejar la opinión por su misma confessión», AGOFM, Collegio sant'Isidoro, 2/39, f. 89r. 
Il tenore degli interventi della Spagna a Roma negli anni a venire rimase sostanzialmente immutato. Sotto il pontificato di Gregorio XV, anche grazie alla favorevole intercessione del cardinal nipote Ludovico Ludovisi, molto vicino ai gesuiti e alla Monarchia spagnola, si arrivò all'emanazione di un decreto del Sant'Uffizio, in data 24 maggio 1622, che andava di nuovo incontro alle richieste degli immacolisti comminando pena di censura a chiunque difendesse l'opinione affermativa non solo in pubblico ma anche nelle controversie e negli scritti privati. Ma appena due mesi dopo (28 luglio, breve Eximii atque singulares) papa Ludovisi concesse ai domenicani il permesso di esporre la tesi macolista nelle loro private dispute, a condizione che non fossero presenti degli esterni. ${ }^{66}$ Filippo IV ebbe dunque motivi per non ritenersi soddisfatto e le missioni andarono avanti, anche se il pontificato barberiniano rappresentò una parentesi negativa per la causa della definizione dogmatica.

Nel 1653 fu nominato ambasciatore straordinario al cospetto di Innocenzo X Pamphili Pedro de Urbina, arcivescovo di Valencia. ${ }^{67}$ Nell'istruzione che questi ricevette al momento della sua partenza il regalismo di cui si parlava poc'anzi risulta addirittura accentuato. II documento si apre con una affermazione che esprime perfettamente i sottintesi più profondi della Monarchia confessionale degli Asburgo: il re di Spagna - si legge - ha deciso di intervenire in una questione di carattere dottrinale come quella dell'Immacolata per difendere la Chiesa di Roma; perché se Roma non definisce una volta per tutte si creano in Spagna occasioni gravissime di scandalo, sia nello stato ecclesiastico, sia in quello secolare. Questa dimensione sociale delle dispute, già presente nella controversia de auxiliis, nel caso dell'Immacolata concezione raggiunse l'apice, entrando definitivamente a far parte dell'ordine del giorno delle questioni dibattute in sede diplomatica. I difensori della sentenza negativa continuavano infatti a non reputare sufficiente il divieto imposto ai domenicani di difendere pubblicamente le proprie posizioni anti-immacoliste, perché questi frati potevano continuare a trattare questa materia «entre si mismos», e di conseguenza era inevitabile che prima o poi sarebbero sorti scandali a causa di questa «conferencia y comunicación de unos con otros sobre este punto», ${ }^{68}$ a sottolineare ancora una volta l'importanza della sociabilità erudita ai fini della sedimentazione di dottrine "nuove" che diventavano agli occhi di tutti accettabili, e quindi ortodosse, sulla base della semplice loro circolazione.

A essere in questione non era tanto la conformità di una dottrina a questo o a quel Padre della Chiesa; in questione, semmai, era la storia della Chiesa, anche recente e recentissima, con un profluvio di autorità messe in campo a sostegno delle proprie tesi (decisioni pontificie da Sisto IV in poi, concilio di Trento, ecc.), e elemento del tutto nuovo e probabilmente peculiare della storia dei rapporti tra Roma e la Monarchia spagnola - il sentire popolare (anche in prospettiva storica) che assurgeva ad autorità di livello equivalente rispetto alle altre. In riferimento al decreto del Sant'Uffizio romano del 20 gennaio 1644, sotto il pontificato di Urbano VIII, che proibiva di attribuire il titolo di "immacolata" alla concezione della Vergine,

${ }^{66}$ Cfr. Pastor, L. von. 1931. Storia dei papi dalla fine del Medioevo: XIII-85, Roma, Désclée.

${ }^{67}$ Cfr. Callado Estela, E. 2011. Tiempos de incienso y pólvora. El arzobispo fray Pedro de Urbina. Valencia, Biblioteca Valenciana.

68 Instrucción real al arzobispo Urbina, AGS, E 3110 e MAE, Ms. 12, pubblicato in Gutiérrez, C. 1955. "España por el dogma de la Inmaculada. La embajada a Roma de 1659. La bula «Sollicitudo» de Alejandro VII", Miscellanea Comillas XXIV: 87. 
Filippo IV metteva in risalto nella sua istruzione a Urbina l'inconciliabilità di tale decreto con le disposizioni apostoliche dettate nel corso del tempo dal papa stesso e con lo scandalo provocato da una decisione che metteva in dubbio una cosa «asentada constantemente en los ánimos de todos los fieles».

Per la Corona spagnola la Chiesa non sa difendere se stessa contro i novatori, e dunque serve qualcuno che si assuma il compito di indicare alla Chiesa la direzione da seguire. L'opinione dei fedeli diventa in tale ottica una autorità nella misura in cui in essa viene recepita e in un certo senso preservata la tradizione ecclesiastica: una sorta di baluardo naturale contro le innovazioni dottrinali, secondo una interpretazione piuttosto osée - e in chiave anticuriale - della tradizione ecclesiastica così come era stata definita dal concilio di Trento, ossia trasmissione orale del lascito della fede sulla scorta del passo dell'epistola ai Romani («fides ex auditu, auditus autem per verbum Christi», Rom. 10,17). Per il bene della religione cattolica è assolutamente necessaria la conformità nella dottrina, perché in caso contrario a farne le spese sarebbe lo stesso principio del primato della Sede Petrina:

Sería gran absurdo que se pudiese llamar y se llamase, como se hace, la Concepción inmaculada en España, Alemania y Francia y en todo el resto de la Iglesia cathólica, y no en Italia ni en Roma. Antes de aquí sería gravísimo el perjuicio de la sentencia pía, tan favorecida siempre de la Sede apostólica; y mucho mayor que si sucediera al contrario, por la veneración de la Santa Sede, como se ha ya dicho, y porque, quando se haga distinción entre el sucesor de san Pedro en el sumo pontificado y el obispo de Roma, aunque algunos theólogos quieren que estén unidas estas dignidades por derecho eclesiástico, otros y los más dicen que, o son una misma, o están unidas por derecho divino, y assí tampoco puede errar la misma Iglesia Romana en quanto tal. ${ }^{69}$

Si arrivava, per mezzo di contorti percorsi concettuali, persino a mettere in dubbio il ruolo esclusivo di Roma nella definizione dell'ortodossia dottrinale: se Roma non può sbagliare, allora deve accettare - per evitare la sua rovina - che altri le indichino la verità, le indichino la strada da seguire per tornare sui suoi passi. Interpretazione davvero singolare della missione della difesa della fede assunta dal re di Spagna. Le pesanti ingerenze volte a ottenere dal papa ciò che egli non intendeva concedere (la definizione dottrinale) subirono come si può vedere una notevole amplificazione nel passaggio dalla disputa de auxiliis a quella sull'Immacolata. L'abilità, non solo retorica, stava nel farle apparire come atti necessari proprio per difendere la Santa Sede da chi disobbediva ai suoi ordini.

Risultato finale dell'ambasciata straordinaria del 1659 fu la bolla Sollicitudo omnium ecclesiarum, emanata da Alessandro VII Chigi l'8 dicembre 1661, che rinnovava i decreti favorevoli all'Immacolata emessi da Sisto IV, da Paolo V e da Gregorio XV, ma che proibiva al contempo ai sostenitori della opinione pia di bollare gli avversari come eretici. ${ }^{70}$ Essa fu l'ultimo importante atto pontificio prima della decisione di Clemente XI, nel 1708, di prescrivere la celebrazione della festa

${ }^{69}$ Ibidem, p. 102.

${ }^{70}$ Cfr. Alfaro, J. 1960. "La Inmaculada Concepción en la Bula «Sollicitudo» a la luz de documentos inéditos", Revista española de teología 20: 5-76; ma soprattutto Gutiérrez, C. 1955. 
dell'Immacolata a tutta la Chiesa e prima che Ludovico Antonio Muratori, alla metà del Settecento, aprisse la nota polemica contro il "voto sanguinario", ossia il voto dei fedeli di difendere «usque ad effusionem sanguinis», se fosse stato necessario, la sentenza dell'Immacolata concezione prima ancora che fosse definita di fede. ${ }^{71}$

Nei tempi lunghissimi dell'evoluzione istituzionale e politica la fondazione sacra della monarchia spagnola fu uno di quei collanti che ressero alla caduta dell'Antico Regime e che si incunearono profondamente nella dialettica tra politica e religione fino al XX secolo. A definizione dogmatica avvenuta i domenicani furono costretti ad allinearsi con il sentimento immacolista così vivo in Spagna, tanto che l'8 dicembre del 1928 fu proprio un domenicano, Luis Urbano, professore di teologia tomista, a pronunciare nel Palazzo Reale di Madrid, di fronte al re Alfonso XIII, un sermone sul rapporto tra la Spagna e l'Immacolata. A un certo punto il predicatore affermò:

Está comprobada, por testimonio de todos los historiógrafos, aquella predilección, humanamente inexplicable, que tuvo la divinidad por ese pueblo de raíces cananeas, que aun persiste en la tierra conservando incólume su raza, sin la defensa de las fronteras ni la savia que brota del suelo nacional: caso único en la historia, para que fuesen los judíos testigos seculares de los oráculos divinos. Cuando esa nación se convirtió de predilecta en deicida y, llevando sobre su frente escrita la maldición de Jehová con la sangra del Justo, fue errante por la tierra dejando por doquier piezas del mosaico deshecho de su unidad política, mandó el Señor a su madre santísima que buscase por el mundo un pueblo donde derramar las ternuras de su corazón, ya que sobre el Vaticano, bajo la tiara de Soberano Pontífice, derramaría la luz de su doctrina con el esplendor de la infalibilidad. Y la Virgen santísima, atraída por el amor y por el dolor, en una escena sublime de bilocación o telecomunicación sobrenatural, pasa el Mediterráneo, y alumbrada por la luna de enero (para que sea inmaculada hasta la luz que le besa en la frente), santifica la tierra española, pisando las riberas del río nacional, del que dio nombre a nuestra madre patria: para que la imagen de la Inmaculada concepción se reflejase ya en la aurora de la Monarquía española, como ideal de su grandeza e inspiradora de sus glorias. $^{72}$

La Spagna come popolo eletto, dopo che il primo popolo eletto, quello ebraico, si era trasformato in deicida e quindi era stato giustamente punito con la diaspora; la Vergine fu dunque inviata da Dio per cercare per il mondo un popolo su cui riversare il proprio amore. Grazie a un fenomeno di bilocazione la Vergine decise di stendere il suo manto divino sulle due sponde del Mediterraneo: su Roma la luce della dottrina, in virtù dell'infallibilità del papa, sulla Spagna la benedizione della Monarchia, a presagio delle sue grandezze future. ${ }^{73}$ In un clima di crescente

${ }^{71}$ Cfr. Le Bachelet, X.-M. 1905. L'Immacolata concezione. Breve storia di un dogma, II-53, Roma, L'Occidente. Sul fronte degli oppositori del Muratori si veda Santi Fiasconaro, F. 2004. II pensiero immacolista di Ignazio Como, OFMConv. († 1774) nella controversia con L. A. Muratori sul "voto sanguinario". Palermo, Officina di Studi Medievali.

72 Sermón pronunciado en la capilla pública del palacio real de Madrid, el día 8 de diciembre de 1928, ante Sus Majestades y Altezas Reales por el m. r. p. fray Luis Urbano o.p., predicador general, doctor en ciencias físicas, lector de teología en el real convento de predicadores de Valencia, miembro de honor de la "Société thomiste" de París, del claustro de doctores de la Universidad pontificia, de la asociación "Francisco de Vitoria", etc, in La Inmaculada concepción y la monarquía española [1929]: 6. Valencia, Ediciones «Rosas y Espinas».

73 II sermone continuava in questo modo: «Aquella estrella de fuego y de sangre que vio Constantino en los aires y que marcaba el crepúsculo vespertino del Imperio romano, señalaba el 
antisemitismo di matrice cattolica ${ }^{74}$ la sacralizzazione dell'identità della Spagna dominata dal regime di Primo de Rivera non poteva risultare più compiuta, ed era altresì in perfetta continuità con il plurisecolare processo di definizione della missione storica della monarchia spagnola, missione di natura sacrale. ${ }^{75}$

\section{BIBLIOGRAFÍA CITADA}

Alfaro, J. 1960. "La Inmaculada Concepción en la Bula «Sollicitudo» a la luz de documentos inéditos", Revista española de teología 20: 5-76.

Aranda Doncel, J. 1999. "Los prebendados del cabildo catedralicio de Córdoba durante los siglos XVI y XVII: la provisión de la canonjía magistral", in A.L. Cortés Peña, M.L. López Guadalupe, Estudios sobre Iglesia y sociedad en Andalucía en la edad moderna: 137-152. Granada: Universidad de Granada

Barrios Aguilera, M. García-Arenal (eds.), 2006. Los plomos del Sacromonte: invención y tesoro, Valencia-Granada-Zaragoza: Universidad de Valencia.

Borromeo, A. 1982. "Il cardinale Cesare Baronio e la Corona spagnola", in R. De Maio, L. Gulia, A. Mazzacane (eds.), Baronio storico e la Controriforma. Atti del Convegno Internazionale di Studi, Sora 6-10 ottobre 1979: 57-166. Sora: Centro di Studi Sorani «Vincenzo Patriarca»

Broggio, P. 2009. La teologia e la politica. Controversie dottrinali, Curia romana e monarchia spagnola tra Cinque e Seicento, Firenze: Leo S. Olschki.

Cabibbo, S. 2003. "Una profetessa alla corte di Spagna. II caso di Maria d'Agreda fra Sei e Settecento", Dimensioni e problemi della ricerca storica 1: 83-105.

Cabibbo, S. 2006, "Vizi e virtù di una «sociedad ensimismada». Maria d'Agreda e la Spagna di Filippo IV", Península. Revista de estudios ibéricos 3: 165-172.

Cabibbo, S. Maria d'Agreda e l'affaire della Mystica ciudad de Dios, Roma, Viella, di prossima pubblicazione.

Callado Estela, E. 2011. Tiempos de incienso y pólvora. El arzobispo fray Pedro de Urbina. Valencia: Biblioteca Valenciana.

Canal M., 1932. "El p. Luis de Aliaga y las controversias teológicas de su tiempo, Archivum Fratrum Praedicatorum 1: 141.

Castellano, J.L. 2008. "La monarchia spagnola come paradigma di una monarchia confessionale", Dimensioni e problemi della ricerca storica:172.

crepúsculo matutino de nuestra nacionalidad: que siempre, en la historia como en los cielos, coincide un ocaso con una aurora. Dio España a Roma la imperial, que se moría, emperadores, generales, soldados, filósofos, sabios, poetas, oradores, artistas; Roma, para agradecerlo, le dio su espléndido derecho, la raigambre de su ciudadanía, la disciplina de sus instituciones armadas y la dulzura de su lengua; y como para que se cumpliese una vez más el pensamiento de los Libros santos, que es necesario sumergir en sangre la túnica de la Iglesia y de la patria para que se torne blanca y hermosa, corrió a torrentes la sangre de los cristianos, y en torno del remanso que se formó en las catacumbas de Zaragoza se abrieron los labios de Prudencio para cantar en estrofas de inmortal lirismo la epopeya de los mártires y la pureza inmaculada de María, a quien llamó con la expresión sublime intemerata Virgo, Virgen inmaculada»; Ibidem, pp. 6-7.

${ }^{74}$ Cfr. Álvarez Chillida, G. 2003. El antisemitismo en España. La imagen del judío (18122002), prólogo de J. Goytisolo: 261-300. Madrid, Marcial Pons.

${ }^{75} \mathrm{Si}$ vedano a tale proposito le considerazioni sviluppate in Aldea, Q. 1998. Religione e politica nella Spagna degli Asburgo, in A. Borromeo (ed.), Storia religiosa della Spagna: 255-286. Milano, Fondazione Ambrosiana Paolo VI. 
Cortés Peña, A.L. 2001. "Andalucía y la Inmaculada Concepción en el siglo XVII", in Id. (ed.), Religión y política durante el Antiguo Régimen: 103-148. Granada: Universidad de Granada.

Feros, A. 2002. El duque de Lerma. Realeza y privanza en la España de Felipe III. Madrid: Marcial Pons

García-Arenal, M. y Rodríguez Mediano, F. 2010. Un Oriente español. Los Moriscos y el Sacromonte en tiempos de Contrarreforma, Madrid: Marcial Pons.

Gutiérrez, C. 1955. "España por el dogma de la Inmaculada. La embajada a Roma de 1659. La bula «Sollicitudo» de Alejandro VII", Miscellanea Comillas XXIV: 87.

Le Bachelet, X.-M. 1905. L'Immacolata concezione. Breve storia di un dogma, II-53, Roma: L'Occidente.

Lopetegui, L. 1946. "La Secretaría de Estado de Paulo V y la composición del «Defensio Fidei» de Suárez", Gregorianum XXVII: 584-596.

Lopetegui, L. 1974. "Renovación y fracaso de las tentativas para obtener una definición dogmática en la Controversia de Auxiliis 1607-1614", Archivo Teológico Granadino 37: 45-81.

López de Madera, G. 1597. Excelencias de la Monarquía y reyno de España: 82 Valladolid: Imprenta de Diego Fernández de Córdova.

Lutz, G. 1973. "Caetani, Antonio", in Dizionario biografico degli Italiani, vol. XVI: 120-125. Roma: Istituto della Enciclopedia Italiana.

Martínez Millán J., Visceglia M.A. (eds.) 2008. La Monarquía de Felipe III: la casa del rey, Madrid: Fundación MAPFRE.

Martínez Medina, F.J., 2006. "Teólogos, religiosidad y magisterio en el Sacromonte de Granada. Los discursos inmaculistas de los libros plúmbeos", in A.L. Cortés Peña (ed.), Poder civil, Iglesia y sociedad en la edad moderna: 295-342. Granada: Universidad de Granada.

Mendia, B. y Artola Arbiza, A.M. 2005. La ven. m. María de Jesús de Ágreda y la Immaculada Concepción: el proceso eclesiástico a la «Mística ciudad de Dios», Ágreda: Concepcionistas Franciscanas.

Olivares, E. 1988. "Juan de Pineda (1557-1637). Biografía. Escritos. Bibliografía", Archivo Teológico Granadino LI: 5-133.

Ollero Pina, J.A. 2003. "Sine labe concepta: conflictos eclesiásticos e ideológicos en la Sevilla de principios del siglo XVII", in C.A. González Sánchez, E. Vila Vilar (eds.), Grafías del Imaginario. Representaciones culturales en España y América (siglos XVI-XVIII): 301-335. México: Fondo de Cultura Económica.

Pastor, L. von. 1931. Storia dei papi dalla fine del Medioevo: XIII-85, Roma: Désclée.

Pou y Martí, J.M. 1931. "Embajadas de Felipe III a Roma pidiendo la definición del dogma de la I. Concepción de María", Archivo ibero-americano XVIII, 34: 381

Prosperi, A. 2006. "L'Immacolata e Siviglia e la fondazione sacra della monarchia spagnola". Studi Storici XLVII: 481-510 [482].

Sánchez Herrero, J. 1985. Las cofradías de Sevilla: historia, antropología, arte, Sevilla: Universidad de Sevilla.

Sánchez Herrero,J. 1992. "Sevilla barroca (1581-1700)", in C. Ros, Historia de la Iglesia de Sevilla: 435. Sevilla: Ed. Castillejo.

Santi Fiasconaro, F. 2004. Il pensiero immacolista di Ignazio Como, OFMConv. ( $\dagger$ 1774) nella controversia con L. A. Muratori sul "voto sanguinario". Palermo: Officina di Studi Medievali 
TEOLOGIA, ORDINI RELIGIOSI E RAPPORTI POLITICI: LA QUESTIONE $2 \mathbf{2 1}$ DELL'IMMACOLATA CONCEZIONE DI MARIA TRA ROMA E MADRID.

(1614-1663)

Scaramella, P., 2008. "«Una materia gravissima, una enorme heresia». Granada, Roma e la controversia sugli apocrifi del Sacromonte". Rivista storica italiana CXX 3: 1003-1044.

Scaramella, P. 2008: 1008. In generale cfr. Pastore, S. 2003. II Vangelo e la spada. L'Inquisizione di Castiglia e i suoi critici (1460-1598): 349 ss Roma: Edizioni di Storia e Letteratura.

Vázquez Lesmes, R., "Pizaño de Palacios, un inmaculista maculado", Boletín de la Real Academia de Córdoba de ciencias, bellas letras y nobles artes: CXXIV (1993): 181-192.

Visceglia, M.A. 2009. Roma e papale e Spagna. Diplomatici, nobili e religiosi tra due corti, Roma: Bulzoni.

Visceglia, M.A. 2007. "Roma e la Monarchia Cattolica nell'età dell'egemonia spagnola in Italia: un bilancio storiografico", in: C.J. Hernando Sánchez (ed.), Roma y España. Un crisol de la cultura europea en la Edad Moderna, Madrid; Sociedad Estatal para la Acción Cultural Exterior.

Williams, P. 2008. "El favorito del rey: Francisco Gómez de Sandoval y Rojas, V marqués de Denia y I duque de Lerma", in J. Martínez Millán, M.A. Visceglia (eds.), La Monarquía de Felipe III: la Corte, vol. III: 185-259. Madrid: MAPFRE 